\title{
Diffraction of flexural-gravity waves by a vertical cylinder of non-circular cross section
}

\author{
N. B. Dişibüyük ${ }^{\mathbf{a}}$, A. A. Korobkin ${ }^{\mathbf{b}}$, O. Yılmaz
}

a Department of Mathematics, Dokuz Eylul University, Izmir, Turkey

b School of Mathematics, University of East Anglia, Norwich, UK

c Department of Mathematics, Izmir Institute of Technology, Izmir, Turkey

\begin{abstract}
The linear three-dimensional problem of flexural-gravity wave (hydro-elastic wave) diffraction by a vertical cylinder of an arbitrary smooth cross section is studied using an asymptotic approach combined with the vertical mode method for water of finite depth. The surface of the water is covered by an infinite, continuous elastic ice plate. The rigid cylinder extends from the sea bottom to the ice surface. The ice plate is frozen to the cylinder. The ice deflection is described by the equation of a thin elastic plate of constant thickness with clamped edge conditions at the cylinder. The flow under the ice is described by the linear theory of potential flows. The coupled problem of wave diffraction is solved in two steps. First, the problem is solved without evanescent waves similar to the problem of water waves diffracted by a vertical cylinder. This solution does not satisfy the edge conditions. Second, a radiation problem with a prescribed motion of the ice plate edge is solved by the vertical mode method. The sum of these two solutions solve the original problem. Both solutions are obtained by an asymptotic method with a small parameter quantifying a small deviation of the cylinder cross section from a circular one. Third-order asymptotic solutions are obtained by solving a set of two-dimensional boundary problems for Helmholtz equations in the exterior of a circle. Strains along the edge, where the ice plate is frozen to the cylinder, are investigated for nearly square and elliptic cross sections of the vertical cylinders depending on the characteristics of ice and incident wave. The strains are shown to be highest in the places of high curvatures of the cross sections. The derived asymptotic formulae can be used in design of vertical columns in ice. They directly relate the strains in ice plate to the shape of the column.
\end{abstract}

Key words: Ice cover, hydro-elastic waves, non-circular vertical cylinder, asymptotic approach, vertical mode method, clamped edge conditions.

\section{Introduction}

Modelling wave propagation through ice covers and interaction of hydro-elastic waves with ocean structures such as floating airports, bridge pylons, semi-submersibles, and tension leg platforms are important for polar engineering. In this paper, hydro-elastic wave diffraction by a vertical cylinder of an arbitrary smooth cross

E-mail addresses: bugurcan.ruzgar@deu.edu.tr (N. B. Dişibüyük), a.korobkin@uea.ac.uk (A. Korobkin), oguzyilmaz@iyte.edu.tr (O. Yilmaz). 
section is investigated. Such a cylinder can be considered as a leg of an offshore platform which is used for exploration of oil and gas from under the seabed.

There are several challenges in diffraction problems of hydro-elastic waves. One of them is that the continuous ice interaction with a structure requires solution of a boundary value problem for Laplace equation with fifth-order derivatives in a boundary condition. Another difficulty is that the eigenfunctions associated with these problems are not orthogonal in the usual sense. The eigenfunction expansion method, known also as vertical mode method, is an efficient direct method to study wave interaction with floating flexible structures. It provides closed form solutions by transforming the corresponding boundary value problems into linear systems of algebraic equations. Fox and Squire [1] applied the eigenfunction expansion method to the interaction of ocean waves propagating from the open ocean with a floating semi-infinite continuous ice sheet. They claimed that the eigenfunctions are complete, but not orthogonal with respect to the usual inner product. Lawrie and Abrahams [2] examined a class of problems including wave equation in which the boundary conditions involve second or higher order normal derivatives. They discovered an orthogonality relation for the eigenfunctions, which involve also some derivatives of these functions. Sahoo et al. [3] introduced a new inner product that satisfies the orthogonality criterion for the eigenfunctions associated with floating flexible plates. They applied the eigenfunction expansion along with this new inner product to study the scattering of flexural-gravity waves by a semi-infinite floating elastic plate. Evans and Porter [4] studied oblique wave scattering by a narrow crack in an ice sheet floating on water of finite depth by using the vertical mode method. They reported that the vertical modes are non-orthogonal in a standard sense, linearly dependent and could be incomplete. They argued that the solution of the problem by the vertical mode method without its validation is questionable. To validate the vertical-mode solution, Evans and Porter [4] solved the same problem by another method based on a Green's function technique and demonstrated that the two solutions are identical. It was reported in [4] that the vertical mode method is much simpler to use than the Green function method.

As an alternative to the vertical mode method, the Weber integral transform in the radial direction was used by Brocklehurst et al. [5] to solve the problem of hydro-elastic wave diffraction by a vertical circular cylinder. The two-dimensional problem of flexural-gravity waves interacting with a vertical wall was studied using a Fourier cosine transform by Brocklehurst et al. [6] and using the vertical-mode method by Bhattacharjee and Soares [7]. The latter approach was generalized to the three-dimensional problems by Korobkin et al. [8] . In this generalised approach, the vertical coordinate is separated in the solution reducing the original three-dimensional problem to a set of two-dimensional diffraction and radiation problems. Each vertical mode corresponds to a root of the dispersion relation for hydro-elastic waves. Such a separation is only possible when both the water depth and the thickness of the ice sheet are constant, the bottom is horizontal and flow region has vertical solid boundaries, see [8]. Korobkin et al. [9] solved the linear three- 
dimensional problem of hydro-elastic wave diffraction from a vertical circular cylinder by both the vertical mode method and the Weber integral transform. They proved that the two solutions are identical for the clamped edge conditions.

The problem of diffraction of hydro-elastic waves by multiple circular cylinders was addressed by Ren et al. [10]. They obtained the solution using the eigenfunction expansions and the Green's second identity repeatedly for domains outside individual cylinders. The wave forces acting on the circular cylinders with clamped and free edge conditions were investigated.

In the present paper, diffraction of hydro-elastic waves by a vertical cylinder of an arbitrary smooth cross section is investigated by using the vertical mode method (Korobkin et al. [8]) and the asymptotic method of Disibuyuk et al. [11]. The asymptotic solution of the problem is based on the idea that the cross section of the cylinder can be described by a perturbation series using a small parameter representing the deviation of the cross section of the cylinder from a circle. This asymptotic method was used successfully by Disibuyuk et al. [11] for the diffraction of water waves by a cylinder of arbitrary smooth cross section. However, a combination of the asymptotic method with the vertical mode method is non-trivial making the hydro-elastic wave diffraction problem much more challenging than the problem of water wave diffraction. The leading order solution corresponds to the problem for an equivalent circular cylinder. Strains along the edge, where the ice plate is frozen to the cylinder, are investigated for nearly square and elliptic cross sections of the vertical cylinders depending on the characteristics of ice and incident wave. The strains are shown to be highest in the places of high curvatures of the cross sections. The derived asymptotic formulae can be used in design of vertical columns in ice. They directly relate the strains in ice plate to the shape of the column.

The outline of the paper is as follows. In section 2, the problem of hydro-elastic wave diffraction by a cylinder of arbitrary smooth cross section is formulated. The ice plate is frozen to the cylinder which is modelled by clamped edge conditions. The problem is decomposed into two parts: one without account for the edge conditions and another one to satisfy the edge conditions. The solution of the first problem is similar to that for water waves. This solution is described in Appendix A. The solution of the second problem is derived in section 3 by combination of the vertical mode method and the asymptotic method. In section 4, asymptotic formula for strain distribution along the contact line is derived. Numerical results for the strain distributions are presented in section 5 for two different geometries: a nearly square and elliptic cylinders. Finally, conclusions are drawn in section 6. 


\section{Formulation of the Problem}

Diffraction of an incident hydro-elastic wave by a vertical cylinder with a non-circular smooth cross section is studied within the linear theory of hydroelasticity. The problem is formulated in a polar coordinate system $(r, \theta, z)$, where the $z$-axis points vertically upwards. The plane $z=-H$ corresponds to the flat rigid bottom, and the plane $z=0$ corresponds to the ice-water interface, where $H$ is water depth. Water surface is covered by an infinite, continuous ice plate which is modelled as a thin elastic plate. The rigid cylinder extends from the sea bottom to the ice-water interface. The problem configuration is shown in Fig. 1.

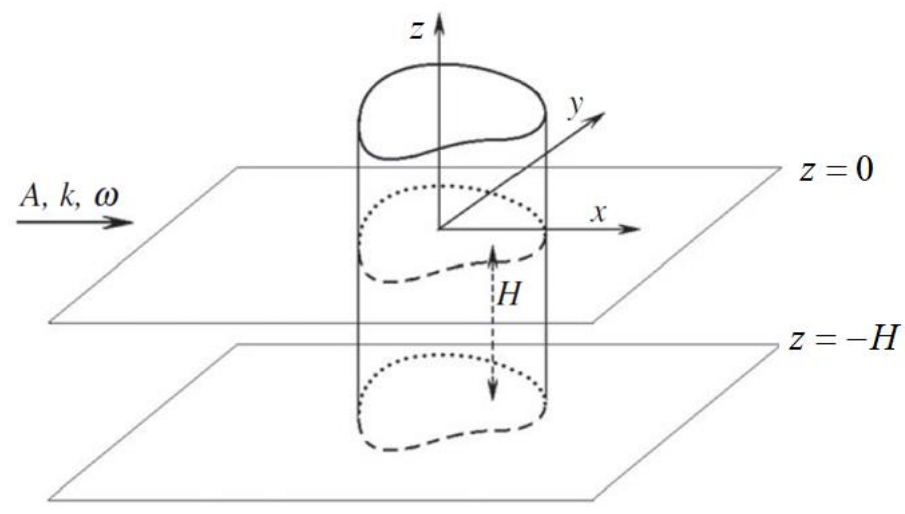

Fig. 1. Problem configuration

The cross section of the vertical cylinder is described by the equation $r=b[1+\varepsilon f(\theta)]$, where $b$ is the mean radius of the cylinder, and $\varepsilon$ is a small non-dimensional parameter of the problem. A smooth and bounded function $f(\theta)$ describes the deviation of the shape of the cylinder from the circular one.

Within the linear hydroelasticity theory, the flow under ice is described by a velocity potential $\phi(r, \theta, z, t)$, which satisfies the Laplace's equation in the flow region,

$$
\nabla^{2} \phi+\phi_{z z}=0 \quad(-H<z<0, \quad r>b[1+\varepsilon f(\theta)]),
$$

where $\nabla^{2} \phi=\phi_{r r}+\frac{1}{r} \phi_{r}+\frac{1}{r^{2}} \phi_{\theta \theta}$. The velocity potential $\phi(r, \theta, z, t)$ satisfies the boundary conditions on the bottom,

$$
\phi_{z}=0 \quad(z=-H, \quad r>b[1+\varepsilon f(\theta)]),
$$

on the vertical surface of the cylinder,

$$
\frac{\partial \phi}{\partial n}=0 \quad(r=b[1+\varepsilon f(\theta)], \quad-H<z<0),
$$

and on the ice-water interface,

$$
\phi_{z}=w_{t}(r, \theta, t) \quad(z=0, \quad r>b[1+\varepsilon f(\theta)]),
$$


where the equation $z=w(r, \theta, t)$ describes the deflection of the ice cover and $\mathbf{n}$ is the unit outward normal vector to the surface of the cylinder, $r=b[1+\varepsilon f(\theta)]$. The deflection $w(r, \theta, t)$ is governed by equation of a thin elastic plate,

$$
m w_{t t}+D \nabla^{4} w=p(r, \theta, 0, t) \quad(r>b[1+\varepsilon f(\theta)]),
$$

where $m=\rho_{i} h_{i}$ is the mass of the ice cover per unit area, $h_{i}$ is the ice thickness, $\rho_{i}$ is the ice density, $D=E h_{i}^{3} /\left[12\left(1-v^{2}\right)\right]$ is the rigidity coefficient for an elastic plate of constant thickness, $E$ is the Young's modulus of the ice, $v$ is the Poisson's ratio and $p$ is the hydrodynamic pressure given on the ice-water interface by the linearized Bernoulli equation,

$$
p(r, \theta, 0, t)=-\rho \phi_{t}(r, \theta, 0, t)-\rho g w(r, \theta, t),
$$

where $\rho$ is the water density and $g$ is the gravitational acceleration.

In addition to the conditions (2) - (4), the condition at the contact line between the ice cover and the surface of the cylinder should be imposed. The conditions at the contact line between the ice cover and the surface of the cylinder where $z=0$ and $r=b[1+\varepsilon f(\theta)]$, can be complicated in practical problems. Here, we assume that the ice cover is frozen to the cylinder which is modelled by the clamped edge conditions,

$$
w=0 \quad \text { and } \quad \frac{\partial w}{\partial n}=0 \quad(r=b[1+\varepsilon f(\theta)]) .
$$

Alternative edge conditions such as simply supported and free edge boundary conditions on the cylinder can also be considered. In the former case, the deflection $w$ and the bending moment $M_{n}$ along the edge should be zero,

$$
w=0 \quad \text { and } \quad M_{n}=-D\left(\frac{\partial^{2} w}{\partial n^{2}}+v \frac{\partial^{2} w}{\partial \tau^{2}}\right)=0 \quad(r=b[1+\varepsilon f(\theta)])
$$

see [12]. The derivatives in (8) are calculated in the direction of normal $n$ and tangent $\tau$ of the curvilinear edge. In the latter case, the edge conditions are given as follows

$$
V_{n}=Q_{n}-\frac{\partial M_{n \tau}}{\partial s}=0 \quad \text { and } M_{n}=0 \quad(r=b[1+\varepsilon f(\theta)]),
$$

where $V_{n}$ is the edge reaction, $Q_{n}$ is the shearing force, $M_{n \tau}$ is the twisting moment and $\partial / \partial s$ denotes the rate of change with respect to arc length parameter $s$. The term $-\frac{\partial M_{n \tau}}{\partial s}$ represents the part of edge reaction due to the distribution of the twisting moment $M_{n \tau}$ along the edge, see [12]. It is seen that the simply supported (8) and free edge conditions (9) are more complicated than the clamped edge conditions (7). The mixed boundary condition, in the case of ice cover is frozen on one portion of the cylinder and free on other 
portion, can be considered as more complicated edge condition. In this case, the boundary conditions for clamped (7) and free (9) edges are combined with angular dependence on the cross section.

In the far-field, the ice plate deflection is represented by a given uni-directional incident wave and waves diffracted from the cylinder.

The flow and ice deflection are caused by an incident hydro-elastic wave,

$$
w_{i n c}(x, t)=A \cos (k x-\omega t),
$$

propagating in the positive $x$-direction (Fig. 1), where $A$ is the amplitude of the incident wave, $k$ is the wave number and $\omega$ is the wave frequency. The real and positive $k$ and $\omega$ in (10) are related by the dispersion relation (Fox and Squire [1])

$$
\omega^{2}\left(m+\frac{\rho}{k \tanh (k H)}\right)=\rho g+D k^{4},
$$

which, for convenience, can be written in the non-dimensional form,

$$
\left(\kappa^{4}+\delta\right) \kappa \tanh (\kappa)-q=0 .
$$

Here, $\kappa=k H$ is the non-dimensional wave number, $q=\left(\omega^{2} H / g\right)\left(H / L_{c}\right)^{4}, \delta=\left(1-\omega^{2} / \omega_{0}^{2}\right)\left(H / L_{c}\right)^{4}$, $L_{c}=(D / \rho g)^{1 / 4}$ is the characteristic length of the ice sheet and $\omega_{0}=(\rho g / m)^{1 / 2}$ is the frequency of broken ice, see [13].

The incident velocity potential $\phi_{i n c}(x, z, t)$ corresponding to the hydro-elastic incident wave (10) reads

$$
\phi_{\text {inc }}(x, z, t)=\frac{A \omega}{k} \frac{\cosh [k(z+H)]}{\sinh (k H)} \sin (k x-\omega t) .
$$

The velocity potential $\phi(r, \theta, z, t)$ and the ice deflection $w(r, \theta, t)$ are the solution of the boundary value problem (1) - (13). Following [9], these functions are decomposed into two parts:

$$
\begin{gathered}
\phi(r, \theta, z, t)=\tilde{\phi}(r, \theta, z, t)+\phi_{c}(r, \theta, z, t), \\
w(r, \theta, t)=\tilde{w}(r, \theta, t)+w_{c}(r, \theta, t),
\end{gathered}
$$

where $\tilde{\phi}(r, \theta, z, t)$ and $\tilde{w}(r, \theta, t)$ correspond to the solution of the problem without accounting for the edge conditions (7). Correspondingly, $\phi_{c}(r, \theta, z, t)$ and $w_{c}(r, \theta, t)$ are correction functions accounting for the conditions (7).

The solution of the hydro-elastic wave diffraction problem without edge conditions includes only the vertical mode presented in the incident wave potential (13) without any evanescent modes corresponding to the complex roots of equation (12). The resulting velocity potential reads 


$$
\tilde{\phi}(r, \theta, z, t)=\frac{A \omega}{k} \frac{\cosh [k(z+H)]}{\sinh (k H)} \operatorname{Re}\left[-i \Phi(r, \theta) e^{-i \omega t}\right]
$$

where Re stands for the real part of a complex number. The form of the velocity potential $\tilde{\phi}(r, \theta, z, t)$ in (16) is similar to that of the solution of the diffraction problem for water waves with the only difference being the dispersive relation (11). The kinematic condition (4) and equation (16) provide the deflection of the ice cover as

$$
\tilde{w}(r, \theta, t)=A \operatorname{Re}\left[\Phi(r, \theta) e^{-i \omega t}\right] .
$$

The velocity potential $\Phi(r, \theta)$ is the solution of the boundary value problem

$$
\begin{gathered}
\nabla^{2} \Phi+k^{2} \Phi=0 \quad(r>b[1+\varepsilon f(\theta)]), \\
\frac{\partial \Phi}{\partial n}=0 \quad(r=b[1+\varepsilon f(\theta)]), \\
\Phi \sim e^{i k r \cos \theta} \quad(r \rightarrow \infty) .
\end{gathered}
$$

The asymptotic method used by Disibuyuk et al. [11] for the diffraction problem of water waves by a cylinder of arbitrary smooth cross section is employed here to find an approximate solution of the problem (18) - (20) as $\varepsilon \rightarrow 0$. The solution procedure is summarized in Appendix A. A similar asymptotic approach is used to determine the correction potential $\phi_{c}(r, \theta, z, t)$, and the correction deflection $w_{c}(r, \theta, t)$.

The correction potential, $\phi_{c}(r, \theta, z, t)$, and the correction deflection, $w_{c}(r, \theta, t)$, satisfy equations (1) - (6), describe outgoing waves at infinity and satisfy the edge conditions,

$$
w_{c}=-A \operatorname{Re}\left[\Phi(b[1+\varepsilon f(\theta)], \theta) e^{-i \omega t}\right] \quad \text { and } \quad \frac{\partial w_{c}}{\partial n}=0 \quad(r=b[1+\varepsilon f(\theta)]),
$$

where $\Phi(r, \theta)$ is the solution of the problem (18) - (20). Using the first condition in (21), the following form for the correction deflection $w_{c}(r, \theta, t)$, where $r>b[1+\varepsilon f(\theta)]$, is suggested,

$$
w_{c}(r, \theta, t)=-A \operatorname{Re}\left[W(r, \theta) e^{-i \omega t}\right] \quad(r>b[1+\varepsilon f(\theta)]),
$$

where the function $W(r, \theta)$ satisfies the conditions,

$$
\begin{gathered}
\frac{\partial W}{\partial n}(b[1+\varepsilon f(\theta)], \theta)=0, \\
W(b[1+\varepsilon f(\theta)], \theta)=\Phi(b[1+\varepsilon f(\theta)], \theta) .
\end{gathered}
$$

Correspondingly the correction potential $\phi_{c}(r, \theta, z, t)$ is sought in the form

$$
\phi_{c}(r, \theta, z, t)=A \omega \operatorname{Re}\left[i \varphi(r, \theta, z) e^{-i \omega t}\right],
$$

where 


$$
\frac{\partial \varphi(r, \theta, 0)}{\partial z}=W(r, \theta)
$$

The potential $\varphi(r, \theta, z)$ satisfies Laplace's equation in the fluid domain,

$$
\nabla^{2} \varphi+\varphi_{z z}=0 \quad(-H<z<0, \quad r>b[1+\varepsilon f(\theta)]) .
$$

The bottom boundary condition (2) and the body boundary condition (3) on the surface of the cylinder give the following conditions,

$$
\begin{gathered}
\frac{\partial \varphi}{\partial z}(r, \theta,-H)=0, \\
\frac{\partial \varphi}{\partial n}(b[1+\varepsilon f(\theta)], \theta, z)=0,
\end{gathered}
$$

respectively. The ice plate equation (5) and the hydrodynamic pressure equation (6) provide the following equation for the function $W(r, \theta)$,

$$
-m \omega^{2} W+D \nabla^{4} W=\rho \omega^{2} \varphi-\rho g W \quad(z=0) .
$$

At infinity, $r \rightarrow \infty$, the correction functions should describe waves radiated from the cylinder. The boundary problem (23) - (30) is solved in the next section by the method of vertical modes for an elastic floating plate and combined with the asymptotic method. After separating the variables in (26) - (30) the vertical mode method reduces the problem to a set of two-dimensional boundary problems, which are solved by the asymptotic method.

\section{Vertical-mode solution}

By the method of separation of variables, the products

$$
\varphi(r, \theta, z)=\varphi_{s}(r, \theta) f_{s}(\hat{z})
$$

satisfy Laplace's equation (27), the boundary conditions on the bottom (28) and ice-water interface (30), and the far-field condition, if $f_{s}(\hat{z})$ are non-trivial solutions of the following spectral problem:

$$
\begin{gathered}
f_{s}^{\prime \prime}(\hat{z})-\kappa_{s}^{2} f_{s}(\hat{z})=0 \quad(-1<\hat{z}<0), \\
\frac{d f_{s}}{d \hat{z}}(-1)=0, \\
\frac{d^{5} f_{s}}{d \hat{z}^{5}}+\delta \frac{d f_{s}}{d \hat{z}}=q f_{s} \quad(\hat{z}=0),
\end{gathered}
$$

where $\hat{z}=z / H, \varphi_{s}(r, \theta)$ satisfy the two-dimensional Helmholtz equation,

$$
\nabla^{2} \varphi_{s}(r, \theta)+\frac{\kappa_{s}^{2}}{H^{2}} \varphi_{s}(r, \theta)=0 \quad(r>b[1+\varepsilon f(\theta)])
$$


which describes out-going waves, and $\kappa_{s}, s \geq-2$, are the roots of the dispersion relation (12), see [8]. The condition (34) is obtained by using condition (26), Laplace's equation (27) and ice plate equation (30). In dispersion relation (12), there are two parameters $\delta$ and $q$, where $q$ is always positive and $\delta$ can be negative or positive. For both positive and negative values of the parameter $\delta$, there are two real roots $\pm \kappa_{0}, \kappa_{0}>0$, four complex roots $\pm a_{0} \pm i b_{0}$, where $a_{0}>0$ and $b_{0}>0$, and infinite number of pure imaginary roots, $\kappa_{n}= \pm i \beta_{n}, \quad \beta_{n+1}>\beta_{n}>0$ for $n \geq 1$ (Fox and Squire [1]). The non-trivial solutions of (32) - (34) normalized with $f_{s}^{\prime}(0)=1$ are given by

$$
f_{s}(\hat{z})=\frac{\cosh \left[\kappa_{s}(1+\hat{z})\right]}{\kappa_{s} \sinh \left(\kappa_{s}\right)}
$$

where we count only the roots $\kappa_{s}$ with non-negative imaginary part, $\kappa_{-2}=-a_{0}+i b_{0}, \kappa_{-1}=a_{0}+i b_{0}, \kappa_{0}>0$ and $\kappa_{s}=i \beta_{s}$ for $s \geq 1$. These solutions are called the vertical modes of a floating elastic plate. The vertical modes are non-orthogonal with respect to a standard integral type inner product but they are orthogonal with respect to the following product,

$$
\langle F(\hat{z}), G(\hat{z})\rangle=\int_{-1}^{0} F(\hat{z}) G(\hat{z}) d \hat{z}+\frac{1}{q}\left[F^{\prime \prime \prime}(0) G^{\prime}(0)+F^{\prime}(0) G^{\prime \prime \prime}(0)\right] .
$$

Then the modes defined by (36) are orthogonal,

$$
\left\langle f_{j}, f_{n}\right\rangle= \begin{cases}0, & j \neq n \\ Q_{n}, & j=n\end{cases}
$$

where

$$
Q_{n}=\frac{1}{2 \kappa_{n}^{2} q^{2}}\left[\kappa_{n}^{2}\left(\kappa_{n}^{4}+\delta\right)^{2}+q\left(5 \kappa_{n}^{4}+\delta-q\right)\right], \quad n=-2,-1,0,1, \ldots
$$

see $[8]$.

The correction potential $\varphi(r, \theta, z)$ is expressed in terms of the vertical modes by using the superposition principle,

$$
\varphi(r, \theta, z)=\sum_{s=-2}^{\infty} \varphi_{s}(r, \theta) f_{s}(z / H)
$$

The kinematic condition (26) provides the correction deflection as

$$
W(r, \theta)=\frac{1}{H} \sum_{s=-2}^{\infty} \varphi_{s}(r, \theta) .
$$

The boundary condition on the cylinder (29) and equation (39) yield 


$$
\frac{\partial \varphi}{\partial n}(b[1+\varepsilon f(\theta)], \theta, z)=\sum_{s=-2}^{\infty} \frac{\partial \varphi_{s}}{\partial n}(b[1+\varepsilon f(\theta)], \theta) f_{s}(z / H)=0 .
$$

Equation (41) does not imply, in general, that

$$
\frac{\partial \varphi_{s}}{\partial n}(b[1+\varepsilon f(\theta)], \theta)=0, \quad s \geq-2, \quad 0 \leq \theta<2 \pi,
$$

because the system of the functions $f_{s}(\hat{z})$ is not necessarily complete and independent. Special care should be taken when calculating the derivatives at the contact line where $r=b[1+\varepsilon f(\theta)]$ and $z=0$. The normal derivative of $\varphi_{s}$ is calculated as,

$$
\frac{\partial \varphi_{s}}{\partial n}=-\frac{H^{3}}{q Q_{s}} \alpha(\theta, \varepsilon), \quad \alpha(\theta, \varepsilon)=\left.\frac{\partial}{\partial n}\left[\nabla^{2} W\right]\right|_{r=b[1+\varepsilon f(\theta)]} \quad(r=b[1+\varepsilon f(\theta)]),
$$

(see Appendix $\mathrm{C}$ ) where $\alpha(\theta, \varepsilon)$ describes the distribution of the shear force along the contact line on the cylinder. The right-hand side of condition (42) can be made independent of the index $s$ by scaling $\varphi_{s}(r, \theta)$ with $-\frac{H^{3}}{q Q_{s}}$. Then we arrive at the following boundary value problems for the scaled potentials $\tilde{\varphi}_{s}(r, \theta)$,

$$
\begin{gathered}
\nabla^{2} \tilde{\varphi}_{s}+\frac{\kappa_{s}^{2}}{H^{2}} \tilde{\varphi}_{s}=0 \quad(r>b[1+\varepsilon f(\theta)]), \\
\frac{\partial \tilde{\varphi}_{s}}{\partial n}=\alpha(\theta, \varepsilon) \quad(r=b[1+\varepsilon f(\theta)]), \\
\lim _{r \rightarrow \infty} r^{1 / 2}\left[\frac{\partial \tilde{\varphi}_{s}}{\partial r}-i \frac{\kappa_{s}}{H} \tilde{\varphi}_{s}\right]=0
\end{gathered}
$$

where $\varphi_{s}(r, \theta)=-\frac{H^{3}}{q Q_{s}} \tilde{\varphi}_{s}(r, \theta), s=-2,-1,0,1, \ldots$ The condition (45) implies out-going waves at infinity. The asymptotic solution to the problem (43) - (45) as $\varepsilon \rightarrow 0$ is obtained in a same way as the solution of the boundary value problem (18) - (20) which is given in Appendix A. However, the body boundary condition (44) is non-homogeneous now. The function $\alpha(\theta, \varepsilon)$ should also be determined. So the solution of the boundary value problem (43) - (45) is more challenging with respect to the problem (18) - (20).

The body boundary condition (44) is written explicitly as

$$
\frac{\partial \tilde{\varphi}_{s}}{\partial n}=\left(\frac{\partial \tilde{\varphi}_{s}}{\partial r}, \frac{1}{r} \frac{\partial \tilde{\varphi}_{s}}{\partial \theta}\right) \cdot\left(\frac{1}{\sqrt{1+\frac{\left[\varepsilon b f^{\prime}(\theta)\right]^{2}}{r^{2}}}}, \frac{1}{r} \frac{-\varepsilon b f^{\prime}(\theta)}{\sqrt{1+\frac{\left[\varepsilon b f^{\prime}(\theta)\right]^{2}}{r^{2}}}}\right)=\alpha(\theta, \varepsilon) \quad(r=b[1+\varepsilon f(\theta)])
$$


which leads to

$$
\frac{\partial \tilde{\varphi}_{s}}{\partial r}(b[1+\varepsilon f(\theta)], \theta)-\frac{\varepsilon f^{\prime}(\theta)}{b[1+\varepsilon f(\theta)]^{2}} \frac{\partial \tilde{\varphi}_{s}}{\partial \theta}(b[1+\varepsilon f(\theta)], \theta)=\alpha(\theta, \varepsilon) \sqrt{1+\left[\frac{\varepsilon f^{\prime}(\theta)}{1+\varepsilon f(\theta)}\right]^{2}} .
$$

Notice that the boundary condition (47) is similar to the boundary condition (A.1), the only difference being the non-homogeneous right-hand side in (47) which is also to be determined. To obtain an asymptotic expansion of the boundary condition (47) as $\varepsilon \rightarrow 0$, the third-order asymptotic expansions of the potentials $\tilde{\varphi}_{n}(r, \theta), n \geq-2$ and the function $\alpha(\theta, \varepsilon)$,

$$
\begin{gathered}
\tilde{\varphi}_{n}(r, \theta)=\tilde{\varphi}_{n 0}(r, \theta)+\varepsilon \tilde{\varphi}_{n 1}(r, \theta)+\varepsilon^{2} \tilde{\varphi}_{n 2}(r, \theta)+\varepsilon^{3} \tilde{\varphi}_{n 3}(r, \theta)+O\left(\varepsilon^{4}\right) \\
\alpha(\theta, \varepsilon)=\alpha_{0}(\theta)+\varepsilon \alpha_{1}(\theta)+\varepsilon^{2} \alpha_{2}(\theta)+\varepsilon^{3} \alpha_{3}(\theta)+O\left(\varepsilon^{4}\right)
\end{gathered}
$$

are used. The resulting approximation of the condition (47) gives the boundary conditions at each asymptotic order,

$\tilde{\varphi}_{n 0, r}(b, \theta)=\alpha_{0}(\theta)$

$\tilde{\varphi}_{n 1, r}(b, \theta)=\tilde{G}_{n 1}(\theta)+\alpha_{1}(\theta)$,

$\tilde{\varphi}_{n 2, r}(b, \theta)=\tilde{G}_{n 2}(\theta)+\frac{1}{2}\left[f^{\prime}(\theta)\right]^{2} \alpha_{0}(\theta)+\alpha_{2}(\theta)$,

$\tilde{\varphi}_{n 3, r}(b, \theta)=\tilde{G}_{n 3}(\theta)-f(\theta)\left[f^{\prime}(\theta)\right]^{2} \alpha_{0}(\theta)+\frac{1}{2}\left[f^{\prime}(\theta)\right]^{2} \alpha_{1}(\theta)+\alpha_{3}(\theta)$,

where

$$
\begin{gathered}
\tilde{G}_{n 1}(\theta)=L_{1 n}\left[\tilde{\varphi}_{n 0}(b, \theta)\right], \\
\tilde{G}_{n 2}(\theta)=L_{1 n}\left[\tilde{\varphi}_{n 1}(b, \theta)\right]+L_{2 n}\left[\tilde{\varphi}_{n 0}(b, \theta)\right], \\
\tilde{G}_{n 3}(\theta)=L_{1 n}\left[\tilde{\varphi}_{n 2}(b, \theta)\right]+L_{2 n}\left[\tilde{\varphi}_{n 1}(b, \theta)\right]+L_{3 n}\left[\tilde{\varphi}_{n 0}(b, \theta)\right],
\end{gathered}
$$

and $L_{j n}, j=1,2,3, n=-2,-1,0, \ldots$ are the differential operators given in (A.8) - (A.10). Note that the boundary conditions (50) - (53) differ from the boundary conditions (A.3) - (A.4) only by the extra terms $\alpha_{i}(\theta), i=0,1,2,3$. It is convenient to represent the functions $\tilde{G}_{n j}(\theta), \alpha_{j}(\theta), j=0,1,2,3$ by their Fourier series,

$$
\tilde{G}_{n j}(\theta)=\sum_{m=0}^{\infty} \frac{\epsilon_{m}}{2}\left[\tilde{G}_{n j m}^{(\mathrm{c})} \cos (m \theta)+\tilde{G}_{n j m}^{(\mathrm{s})} \sin (m \theta)\right]
$$




$$
\alpha_{j}(\theta)=\sum_{m=0}^{\infty} \frac{\epsilon_{m}}{2}\left[\alpha_{j m}^{(c)} \cos (m \theta)+\alpha_{j m}^{(s)} \sin (m \theta)\right]
$$

where $\tilde{G}_{n j m}^{(\mathrm{c})}, \quad \tilde{G}_{n j m}^{(s)}$ are known and $\alpha_{j m}^{(c)}, \quad \alpha_{j m}^{(s)}$ are unknown Fourier coefficients for $j=0,1,2,3$, $m=0,1,2,3, \ldots$ and $\epsilon_{m}$ is the Neumann symbol, $\epsilon_{0}=1, \epsilon_{m}=2$ for $m \geq 1$. The upper indexes "c" and "s" indicate the coefficient of cosine and sine, respectively.

The correction deflection $W(r, \theta)$ is related to the scaled potentials $\tilde{\varphi}_{s}(r, \theta), s \geq-2$ as follows

$$
W(r, \theta)=-\frac{H^{2}}{q} \sum_{s=-2}^{\infty} \frac{\tilde{\varphi}_{s}(r, \theta)}{Q_{s}},
$$

see (40). The asymptotic formula (48) provides

$$
W(r, \theta)=W_{0}(r, \theta)+\varepsilon W_{1}(r, \theta)+\varepsilon^{2} W_{2}(r, \theta)+\varepsilon^{3} W_{3}(r, \theta)+O\left(\varepsilon^{4}\right),
$$

where

$$
W_{j}(r, \theta)=-\frac{H^{2}}{q} \sum_{n=-2}^{\infty} \frac{\tilde{\varphi}_{n j}(r, \theta)}{Q_{n}}, \quad j=0,1,2,3 .
$$

The edge condition (24) and expansions (A.2) and (60) give

$$
\begin{aligned}
& W_{0}(b, \theta)=\Phi_{0}(b, \theta), \\
& W_{1}(b, \theta)=\Phi_{1}(b, \theta),
\end{aligned}
$$

$W_{2}(b, \theta)+b f(\theta) W_{1, r}(b, \theta)+\frac{[b f(\theta)]^{2}}{2} W_{0, r r}(b, \theta)=\Phi_{2}(b, \theta)+b f(\theta) \Phi_{1, r}(b, \theta)+\frac{[b f(\theta)]^{2}}{2} \Phi_{0, r r}(b, \theta)$,

$$
\begin{aligned}
W_{3}(b, \theta)+b f(\theta) W_{2, r}( & b, \theta)+\frac{[b f(\theta)]^{2}}{2} W_{1, r r}(b, \theta)+\frac{[b f(\theta)]^{3}}{6} W_{0, r r r}(b, \theta) \\
& =\Phi_{3}(b, \theta)+b f(\theta) \Phi_{2, r}(b, \theta)+\frac{[b f(\theta)]^{2}}{2} \Phi_{1, r r}(b, \theta)+\frac{[b f(\theta)]^{3}}{6} \Phi_{0, r r r}(b, \theta),
\end{aligned}
$$

where $\Phi_{0}(r, \theta)$ is given by (A.11) and $\Phi_{j}(r, \theta), j=1,2,3$ are given by (A.14). 


\subsection{The leading order correction}

The leading order scaled correction potentials $\tilde{\varphi}_{n 0}(r, \theta), n=-2,-1,0,1, \ldots$, satisfy the boundary value problem,

$$
\begin{gathered}
\nabla^{2} \tilde{\varphi}_{n 0}+\frac{\kappa_{n}^{2}}{H^{2}} \tilde{\varphi}_{n 0}=0 \quad(r>b), \\
\tilde{\varphi}_{n 0, r}(b, \theta)=\alpha_{0}(\theta) \quad(r=b), \\
\lim _{r \rightarrow \infty} r^{1 / 2}\left[\frac{\partial \tilde{\varphi}_{n 0}}{\partial r}-i \frac{\kappa_{n}}{H} \tilde{\varphi}_{n 0}\right]=0,
\end{gathered}
$$

where $\alpha_{0}(\theta)$ is still to be determined. The leading order deflection $W_{0}(r, \theta)$ is given by

$$
W_{0}(r, \theta)=-\frac{H^{2}}{q} \sum_{n=-2}^{\infty} \frac{\tilde{\varphi}_{n 0}(r, \theta)}{Q_{n}} .
$$

The condition (67) and the Fourier series (58) suggest the following formula for $\tilde{\varphi}_{n 0}(r, \theta)$,

$$
\tilde{\varphi}_{n 0}(r, \theta)=\sum_{m=0}^{\infty} \frac{\epsilon_{m}}{2}\left[\alpha_{0 m}^{(c)} \cos (m \theta)+\alpha_{0 m}^{(s)} \sin (m \theta)\right] R_{n m}(r), \quad n=-2,-1,0, \ldots
$$

where

$$
R_{n m}(r)=H \frac{H_{m}^{(1)}\left(\kappa_{n} \hat{r}\right)}{\kappa_{n} H_{m}^{(1)^{\prime}}\left(\kappa_{n} \hat{b}\right)}, \quad \hat{r}=r / H, \quad \hat{b}=b / H
$$

Here $H_{m}^{(1)}(r)$ is the Hankel function of the first kind with order $m$ corresponding to outward-propagating cylindrical waves. Prime stands for derivatives with respect to the argument. The functions $R_{n m}(r), m=0,1,2, \ldots, n=-2,-1,0, \ldots$, are the solutions of the boundary-value problem

$$
r^{2} R_{n m}^{\prime \prime}(r)+r R_{n m}^{\prime}(r)+\left[\frac{\kappa_{n}^{2}}{H^{2}} r^{2}-m^{2}\right] R_{n m}(r)=0 \quad(r>b), \quad R_{n m}^{\prime}(b)=1,
$$

describing waves propagating to infinity from the cylinder.

The unknown coefficients $\alpha_{0 m}^{(c)}, \alpha_{0 m}^{(s)}, m=0,1,2, \ldots$ in (70) are determined using (69), (62) and (A.12). We obtain,

$$
W_{0}(b, \theta)=-\frac{H^{2}}{q} \sum_{n=-2}^{\infty} \frac{\tilde{\varphi}_{n 0}(b, \theta)}{Q_{n}}=
$$




$$
-\frac{H^{2}}{q} \sum_{n=-2}^{\infty} \frac{1}{Q_{n}}\left[\sum_{m=0}^{\infty} \frac{\epsilon_{m}}{2}\left[\alpha_{0 m}^{(c)} \cos (m \theta)+\alpha_{0 m}^{(s)} \sin (m \theta)\right] R_{n m}(b)\right]=\frac{2 i}{\pi k b} \sum_{m=0}^{\infty} \frac{\epsilon_{m} i^{m}}{H_{m}^{(1)^{\prime}}(k b)} \cos (m \theta) .
$$

Changing the order of the summation we get

$$
-\frac{H^{2}}{q} \sum_{m=0}^{\infty}\left\{\frac{\epsilon_{m}}{2}\left[\alpha_{0 m}^{(c)} \cos (m \theta)+\alpha_{0 m}^{(s)} \sin (m \theta)\right]\left(\sum_{n=-2}^{\infty} \frac{R_{n m}(b)}{Q_{n}}\right)\right\}=\frac{2 i}{\pi k b} \sum_{m=0}^{\infty} \frac{\epsilon_{m} i^{m}}{H_{m}^{(1)^{\prime}}(k b)} \cos (m \theta),
$$

which gives

$$
\alpha_{0 m}^{(c)}=\frac{-4 q}{\pi k b H^{2}} \frac{i^{m+1} g_{m}}{H_{m}^{(1)^{\prime}}(k b)}, \quad m=0,1,2,3 \ldots
$$

and $\alpha_{0 m}^{(s)}=0, m=1,2,3 \ldots$, where

$$
g_{m}=\left[\sum_{n=-2}^{\infty} \frac{R_{n m}(b)}{Q_{n}}\right]^{-1}, m=0,1,2,3 \ldots
$$

It is seen that the leading order potentials $\tilde{\varphi}_{n 0}(r, \theta), n=-2,-1,0,1, \ldots$, correspond to the solution of the diffraction problem of hydro-elastic waves by a vertical circular cylinder (see, [9]).

\subsection{The first-order correction}

The first-order scaled correction potentials, $\tilde{\varphi}_{n 1}(r, \theta), n=-2,-1,0,1, \ldots$, satisfy the boundary value problems,

$$
\begin{gathered}
\nabla^{2} \tilde{\varphi}_{n 1}+\frac{\kappa_{n}^{2}}{H^{2}} \tilde{\varphi}_{n 1}=0 \quad(r>b), \\
\tilde{\varphi}_{n 1, r}(b, \theta)=\tilde{G}_{n 1}(\theta)+\alpha_{1}(\theta), \\
\lim _{r \rightarrow \infty} r^{1 / 2}\left[\frac{\partial \tilde{\varphi}_{n 1}}{\partial r}-i \frac{\kappa_{n}}{H} \tilde{\varphi}_{n 1}\right]=0,
\end{gathered}
$$

which are similar to (66) - (68) but with extra terms in (75). These terms, $\tilde{G}_{n 1}(\theta)$, are given functions. The function $\alpha_{1}(\theta)$ is to be determined. The first-order deflection $W_{1}(r, \theta)$ is given by

$$
W_{1}(r, \theta)=-\frac{H^{2}}{q} \sum_{n=-2}^{\infty} \frac{\tilde{\varphi}_{n 1}(r, \theta)}{Q_{n}} .
$$

Using the boundary condition (75) and the Fourier series (57), (58), the potentials $\tilde{\varphi}_{n 1}(r, \theta)$, $n=-2,-1,0,1, \ldots$, are obtained as 


$$
\tilde{\varphi}_{n 1}(r, \theta)=\sum_{m=0}^{\infty} \frac{\epsilon_{m}}{2}\left\{\left[\tilde{G}_{n 1 m}^{(c)}+\alpha_{1 m}^{(c)}\right] \cos (m \theta)+\left[\tilde{G}_{n 1 m}^{(s)}+\alpha_{1 m}^{(s)}\right] \sin (m \theta)\right\} R_{n m}(r) .
$$

To determine the unknown coefficients $\alpha_{1 m}^{(c)}, \alpha_{1 m}^{(s)}, m=0,1,2, \ldots$ in (78), the formula (77) and the condition (63) are used, where $\Phi_{1}(b, \theta)$ is given by (A.14),

$$
\begin{aligned}
W_{1}(b, \theta)=-\frac{H^{2}}{q} \sum_{n=-2}^{\infty} \frac{1}{Q_{n}}\left[\sum _ { m = 0 } ^ { \infty } \frac { \epsilon _ { m } } { 2 } \left\{\left[\tilde{G}_{n 1 m}^{(c)}+\alpha_{1 m}^{(c)}\right] \cos (m \theta)\right.\right. & \left.\left.+\left[\tilde{G}_{n 1 m}^{(s)}+\alpha_{1 m}^{(s)}\right] \sin (m \theta)\right\} R_{n m}(b)\right] \\
& =\sum_{m=0}^{\infty} \frac{\epsilon_{m}}{2}\left[G_{1 m}^{(\mathrm{c})} \cos (m \theta)+G_{1 m}^{(\mathrm{s})} \sin (m \theta)\right] R_{0 m}(b) .
\end{aligned}
$$

Changing the order of the summation in the above equation we get

$$
\begin{aligned}
& -\frac{H^{2}}{q} \sum_{m=0}^{\infty} \frac{\epsilon_{m}}{2}\left[\alpha_{1 m}^{(c)} \cos (m \theta)+\alpha_{1 m}^{(s)} \sin (m \theta)\right]\left[\sum_{n=-2}^{\infty} \frac{R_{n m}(b)}{Q_{n}}\right] \\
& -\frac{H^{2}}{q} \sum_{m=0}^{\infty} \frac{\epsilon_{m}}{2}\left\{\left[\sum_{n=-2}^{\infty} \frac{R_{n m}(b)}{Q_{n}} \tilde{G}_{n 1 m}^{(c)}\right] \cos (m \theta)+\left[\sum_{n=-2}^{\infty} \frac{R_{n m}(b)}{Q_{n}} \tilde{G}_{n 1 m}^{(s)}\right] \sin (m \theta)\right\} \\
& =\sum_{m=0}^{\infty} \frac{\epsilon_{m}}{2}\left[G_{1 m}^{(\mathrm{c})} \cos (m \theta)+G_{1 m}^{(\mathrm{s})} \sin (m \theta)\right] R_{0 m}(b),
\end{aligned}
$$

which gives

$$
\begin{aligned}
& \alpha_{1 m}^{(c)}=g_{m}\left\{-\frac{q}{H^{2}} R_{0 m}(b) G_{1 m}^{(c)}-\tilde{g}_{1 m}^{(c)}\right\}, \quad m=0,1,2, \ldots, \\
& \alpha_{1 m}^{(s)}=g_{m}\left\{-\frac{q}{H^{2}} R_{0 m}(b) G_{1 m}^{(s)}-\tilde{g}_{1 m}^{(s)}\right\}, \quad m=1,2, \ldots,
\end{aligned}
$$

where $g_{m}$ is given in (73), $G_{1 m}^{(c)}, G_{1 m}^{(s)}$ are defined in (A.13), $\tilde{G}_{n 1 m}^{(c)}, \tilde{G}_{n 1 m}^{(s)}$ are defined in (57) and

$$
\tilde{g}_{j m}^{(c)}=\left[\sum_{n=-2}^{\infty} \frac{R_{n m}(b)}{Q_{n}} \tilde{G}_{n j m}^{(c)}\right], \quad \tilde{g}_{j m}^{(s)}=\left[\sum_{n=-2}^{\infty} \frac{R_{n m}(b)}{Q_{n}} \tilde{G}_{n j m}^{(s)}\right], \quad j=1,2,3, \ldots
$$

\subsection{Higher order correction}

The boundary value problem (66) - (68) for the leading order potentials $\tilde{\varphi}_{n 0}(r, \theta)$, and the boundary value problem (74) - (76) for the first order potentials $\tilde{\varphi}_{n 1}(r, \theta)$ are similar with the only change being in the body boundary conditions (67) and (75). The higher order potentials $\tilde{\varphi}_{n j}(r, \theta), j=2,3$ satisfy similar boundary value problems as (74) - (76) but with more known terms in the body boundary condition (see Appendix D). 
Using $\alpha_{j m}^{(c)}, \alpha_{j m}^{(s)}, j=0,1,2,3, m=0,1,2, \ldots$, the correction potential and deflection up to the order $O\left(\varepsilon^{4}\right)$ are given as follows,

$$
\begin{aligned}
\varphi(r, \theta, z) & =\sum_{n=-2}^{\infty} \varphi_{n}(r, \theta) f_{n}(z / H)=-\frac{H^{3}}{q} \sum_{n=-2}^{\infty} \frac{1}{Q_{n}} \tilde{\varphi}_{n}(r, \theta) f_{n}(z / H) \\
& =-\frac{H^{3}}{q} \sum_{n=-2}^{\infty} \frac{1}{Q_{n}}\left[\tilde{\varphi}_{n 0}(r, \theta)+\varepsilon \tilde{\varphi}_{n 1}(r, \theta)+\varepsilon^{2} \tilde{\varphi}_{n 2}(r, \theta)+\varepsilon^{3} \tilde{\varphi}_{n 3}(r, \theta)+O\left(\varepsilon^{4}\right)\right] f_{n}(z / H) \\
& =-\frac{H^{3}}{q} \sum_{n=-2}^{\infty} \frac{1}{Q_{n}}\left[\sum_{m=0}^{\infty} \frac{\epsilon_{m}}{2}\left[\left(\sum_{j=0}^{3} \varepsilon^{j} \mu_{j m}^{(c)}\right) \cos (m \theta)+\left(\sum_{j=0}^{3} \varepsilon^{j} \mu_{j m}^{(s)}\right) \sin (m \theta)+O\left(\varepsilon^{4}\right)\right] R_{n m}(r)\right] f_{n}(z / H) \\
& =-\frac{H^{3}}{q} \sum_{m=0}^{\infty} \frac{\epsilon_{m}}{2}\left[\left(\sum_{j=0}^{3} \varepsilon^{j} \mu_{j m}^{(c)}\right) \cos (m \theta)+\left(\sum_{j=0}^{3} \varepsilon^{j} \mu_{j m}^{(s)}\right) \sin (m \theta)+O\left(\varepsilon^{4}\right)\right]\left(\sum_{n=-2}^{\infty} \frac{f_{n}(z / H)}{Q_{n}} R_{n m}(r)\right),
\end{aligned}
$$

and

$$
\begin{aligned}
W(r, \theta) & =\frac{1}{H} \sum_{n=-2}^{\infty} \varphi_{n}(r, \theta)=-\frac{H^{2}}{q} \sum_{n=-2}^{\infty} \frac{1}{Q_{n}} \tilde{\varphi}_{n}(r, \theta) \\
& =-\frac{H^{2}}{q} \sum_{n=-2}^{\infty} \frac{1}{Q_{n}}\left[\tilde{\varphi}_{n 0}(r, \theta)+\varepsilon \tilde{\varphi}_{n 1}(r, \theta)+\varepsilon^{2} \tilde{\varphi}_{n 2}(r, \theta)+\varepsilon^{3} \tilde{\varphi}_{n 3}(r, \theta)+O\left(\varepsilon^{4}\right)\right] \\
& =-\frac{H^{2}}{q} \sum_{n=-2}^{\infty} \frac{1}{Q_{n}}\left[\sum_{m=0}^{\infty} \frac{\epsilon_{m}}{2}\left[\left(\sum_{j=0}^{3} \varepsilon^{j} \mu_{j m}^{(c)}\right) \cos (m \theta)+\left(\sum_{j=0}^{3} \varepsilon^{j} \mu_{j m}^{(s)}\right) \sin (m \theta)+O\left(\varepsilon^{4}\right)\right] R_{n m}(r)\right] \\
& =-\frac{H^{2}}{q} \sum_{m=0}^{\infty} \frac{\epsilon_{m}}{2}\left[\left(\sum_{j=0}^{3} \varepsilon^{j} \mu_{j m}^{(c)}\right) \cos (m \theta)+\left(\sum_{j=0}^{3} \varepsilon^{j} \mu_{j m}^{(s)}\right) \sin (m \theta)+O\left(\varepsilon^{4}\right)\right]\left(\sum_{n=-2}^{\infty} \frac{R_{n m}(r)}{Q_{n}}\right)
\end{aligned}
$$

where $\mu_{j m}^{(c)}=\left\{\tilde{G}_{n j m}^{(c)}+\gamma_{(j-1) m}^{(c)}+\alpha_{j m}^{(c)}\right\}, \mu_{j m}^{(s)}=\left\{\tilde{G}_{n j m}^{(s)}+\gamma_{(j-1) m}^{(s)}+\alpha_{j m}^{(s)}\right\}, j=0,1,2,3, m=0,1,2, \ldots$ such that $\gamma_{-1 m}^{(c)}$, $\gamma_{-1 m}^{(s)}, \gamma_{0 m}^{(c)}, \gamma_{0 m}^{(s)}$ and $\tilde{G}_{n 0 m}^{(c)}, \tilde{G}_{n 0 m}^{(s)}$ are zero.

In summary, in order to solve the diffraction problem (1) - (7), (10) - (13) of hydro-elastic waves from a non-circular cylinder, the vertical-mode method combined with an asymptotic approach is used and at each asymptotic order either a diffraction or a radiation problem for a circular cylinder in ice is obtained: the leading order problem (66) - (68) corresponds to the diffraction problem and the higher order problems correspond to the radiation problems.

The hydro-elastic wave diffraction problem (1) - (7), (10) - (13) is solved under the assumption of clamped edge conditions (7). It should be noted that the present method can also be used to solve the problem with different edge conditions. There will be no increase in difficulty to solve the problem with simply supported (8) or free edge (9) conditions. But, the problem with mixed boundary conditions where ice cover is frozen 
on one portion of the cylinder and free on other portion, is more challenging because of the angular dependence of the conditions at the cross section. Note that the solution procedure for different edge conditions will be the same as the one with clamped edge conditions, see [9]. Specifically the conditions (23), (24), (44) and (62) - (65) will be different for different edge conditions.

\section{Strain Distribution along the contact line at the Vertical Cylinder with Nearly Circular Cross Section}

Due to the wave-structure interaction, the ice surrounding the structure can be broken. To investigate the possibility of the ice breaking it is necessary to calculate the strain distribution around the cylinder in waves. In this paper, we investigate only the strains along the contact line, where the ice plate is clamped to the vertical cylinder. The yield strain of the ice, the maximum value of the strain at which ice begins to deform plastically, is estimated as $8 \times 10^{-5}$, see [5]. On the contact line between the cylinder surface and the ice cover, only the normal strain component, $\tau_{n}(\theta, t)=\frac{h_{i}}{2} \frac{\partial^{2} w}{\partial n^{2}}(b[1+\varepsilon f(\theta)], \theta, t)$, is not equal to zero, where $\mathbf{n}=\left(n_{1}(\theta), n_{2}(\theta)\right)$ is the unit outward normal vector to the surface of the cylinder $r=b[1+\varepsilon f(\theta)]$. The second normal derivative of the deflection, $\frac{\partial^{2} w}{\partial n^{2}}(r, \theta, t)$, at the contact line is calculated using the definition of normal derivative and the formulae for the elements $n_{1}$ and $n_{2}$ of the normal vector from (46)

$$
\begin{aligned}
\frac{\partial^{2} w}{\partial n^{2}}(b[1+\varepsilon f(\theta)], \theta, t) & =\frac{\partial^{2} w}{\partial r^{2}} n_{1}^{2}+\frac{2}{r}\left[\frac{\partial^{2} w}{\partial r \partial \theta}-\frac{1}{r} \frac{\partial w}{\partial \theta}\right] n_{1} n_{2}+\frac{1}{r}\left[\frac{\partial w}{\partial r}+\frac{1}{r} \frac{\partial^{2} w}{\partial \theta^{2}}\right] n_{2}^{2} \\
& =\frac{1}{n_{1}^{2}(\theta)} \frac{\partial^{2} w}{\partial r^{2}}(b[1+\varepsilon f(\theta)], \theta, t) .
\end{aligned}
$$

To prove (82), we shall show first that the terms with the first derivatives in (82) cancel each other for the clamped conditions (7) at the contact line. These conditions provide

$$
\begin{gathered}
w(b[1+\varepsilon f(\theta)], \theta, t)=0 \\
n_{1} \frac{\partial w}{\partial r}(b[1+\varepsilon f(\theta)], \theta, t)+\frac{n_{2}}{r} \frac{\partial w}{\partial \theta}(b[1+\varepsilon f(\theta)], \theta, t)=0 .
\end{gathered}
$$

Differentiating the equality (83) in $\theta$ and combining the result with the equality (84), we obtain

$$
\begin{aligned}
& \varepsilon b f^{\prime}(\theta) \frac{\partial w}{\partial r}+\frac{\partial w}{\partial \theta}=0 \\
& \frac{\partial w}{\partial r}-\frac{\varepsilon b f^{\prime}(\theta)}{r^{2}} \frac{\partial w}{\partial \theta}=0
\end{aligned}
$$


which gives

$$
\frac{\partial w}{\partial r}(b[1+\varepsilon f(\theta)], \theta, t)=0, \quad \frac{\partial w}{\partial \theta}(b[1+\varepsilon f(\theta)], \theta, t)=0 .
$$

Therefore, both $\partial w / \partial \theta$ and $\partial w / \partial r$ in (82) are equal to zero. Differentiating equations (87) in $\theta$, we obtain the following relations between the second derivatives of the deflection at the contact line

$$
\begin{aligned}
& \varepsilon b f^{\prime}(\theta) \frac{\partial^{2} w}{\partial r^{2}}+\frac{\partial^{2} w}{\partial r \partial \theta}=0, \\
& \varepsilon b f^{\prime}(\theta) \frac{\partial^{2} w}{\partial r \partial \theta}+\frac{\partial^{2} w}{\partial \theta^{2}}=0,
\end{aligned}
$$

where $r=b[1+\varepsilon f(\theta)]$. Substituting the relations (88) - (89) in the formula for $\partial^{2} w / \partial n^{2}$, we arrive at the formula (82). Hence, the second normal derivative of the deflection at the contact line with clamped edge conditions is proportional to the second derivative of the deflection in the radial direction.

The complete deflection of the ice plate, $w(r, \theta, t)$, see (15), is given by its asymptotic expansion

$$
\begin{aligned}
w(r, \theta, t) & =\tilde{w}(r, \theta, t)+w_{c}(r, \theta, t) \\
& =w_{0}(r, \theta, t)+\varepsilon w_{1}(r, \theta, t)+\varepsilon^{2} w_{2}(r, \theta, t)+\varepsilon^{3} w_{3}(r, \theta, t)+O\left(\varepsilon^{4}\right),
\end{aligned}
$$

where

$$
w_{j}(r, \theta, t)=A \operatorname{Re}\left[\left\{\Phi_{j}(r, \theta)-W_{j}(r, \theta)\right\} e^{-i \omega t}\right], \quad j=0,1,2,3,
$$

and $\Phi_{j}(r, \theta)$ are given by formulas (A.11), (A.14) and $W_{j}(r, \theta)$ are given by the formula (61) for $j=0,1,2,3,4$. Substituting the third-order asymptotic expansion (90) of the deflection $w(r, \theta, t)$ into (82) gives the asymptotic expansion of $\frac{\partial^{2} w}{\partial n^{2}}(r, \theta, t)$ on cylinder surface,

$$
\begin{aligned}
\frac{\partial^{2} w}{\partial n^{2}}(b[1+\varepsilon f(\theta)], \theta, t)= & w_{0, r r}(b, \theta, t) \\
& +\varepsilon\left[b f(\theta) w_{0, r r r}(b, \theta, t)+w_{1, r r}(b, \theta, t)\right] \\
+ & \varepsilon^{2}\left[\left[f^{\prime}(\theta)\right]^{2} w_{0, r r}+\frac{b^{2} f^{2}(\theta)}{2} w_{0, r r r r}+b f(\theta) w_{1, r r r}+w_{2, r r}\right] \\
+ & {\left[\begin{array}{l}
-2 f(\theta)\left[f^{\prime}(\theta)\right]^{2} w_{0, r r}+b f(\theta)\left[f^{\prime}(\theta)\right]^{2} w_{0, r r r} \\
+\frac{1}{6} b^{3} f^{3}(\theta) w_{0, r r r r}+\left[f^{\prime}(\theta)\right]^{2} w_{1, r r}+\frac{1}{2} b^{2} f^{2}(\theta) w_{1, r r r} \\
+b f(\theta) w_{2, r r r}+w_{3, r r}
\end{array}\right]+O\left(\varepsilon^{4}\right), }
\end{aligned}
$$

where the derivatives of the $w_{j}(r, \theta, t), j=0,1,2,3$ are calculated at $r=b$. 


\section{Numerical Results}

The hydro-elastic behaviour of the ice cover around a nearly square and an elliptic cylinder is investigated by using the asymptotic expansion (91) for the normal strain component $\tau_{n}(\theta, t)=\frac{h_{i}}{2} \frac{\partial^{2} w}{\partial n^{2}}(b[1+\varepsilon f(\theta)], \theta, t)=\operatorname{Re}\left[E(\theta) e^{-i \omega t}\right]$, where $|E(\theta)|$ is the amplitude of this strain along the contact line between the ice and the cylinder. Calculations are performed for an incident linear wave of amplitude $A=0.01 \mathrm{~m}$, for a sea ice with density $\rho_{i}=917 \mathrm{~kg} / \mathrm{m}^{3}$, thickness $h_{i}=0.2,0.5$ and $1 \mathrm{~m}$, Young's modulus $E=4.2 \times 10^{9} \mathrm{~N} / \mathrm{m}^{2}$, Poisson's ratio $v=0.33$. The water density is $\rho=1026 \mathrm{~kg} / \mathrm{m}^{3}$, water depth is $H=15 \mathrm{~m}$. These particular values were used by Korobkin et al. [9] in their studies.

\subsection{Strain Distribution on a Nearly Square Cylinder}

In this section, a vertical cylinder with nearly square cross section is considered. Let the equation $r=a C(\theta)$ describe the square with side length $2 a$ in polar coordinates, where

$$
C(\theta)= \begin{cases}\frac{1}{\cos (\theta)}, & 0 \leq \theta \leq \frac{\pi}{4}, \\ \frac{1}{\sin (\theta)}, & \frac{\pi}{4} \leq \theta \leq \frac{3 \pi}{4}, \\ \frac{-1}{\cos (\theta)}, & \frac{3 \pi}{4} \leq \theta \leq \frac{5 \pi}{4}, \\ \frac{-1}{\sin (\theta)}, & \frac{5 \pi}{4} \leq \theta \leq \frac{7 \pi}{4}, \\ \frac{1}{\cos (\theta)}, & \frac{7 \pi}{4} \leq \theta \leq 2 \pi .\end{cases}
$$

First the Fourier coefficients of $C(\theta), 0 \leq \theta \leq 2 \pi$, are determined, and then the corresponding Fourier series is converted into the form $r=b[1+\varepsilon f(\theta)]$, identifying the values of $b, \varepsilon$, and the function $f(\theta)$. Then, the asymptotic formula (91) is used to calculate the amplitude of the normal strain component $|E(\theta)|$. A square has four lines of symmetry, so the Fourier series of the function $C(\theta)$ contains only terms $\cos (4 m \theta)$, $m \geq 0$ :

$$
C(\theta)=\frac{1}{2} C_{0}+\sum_{m=1}^{\infty} C_{4 m} \cos (4 m \theta)
$$

where 


$$
C_{m}=\frac{8}{\pi} \int_{0}^{\pi / 4} C(\theta) \cos (4 m \theta) \mathrm{d} \theta=\frac{8}{\pi} \int_{0}^{\pi / 4} \frac{\cos (4 m \theta)}{\cos (\theta)} \mathrm{d} \theta
$$

and

$C_{0}=2.2444, C_{4}=-0.156444, C_{8}=0.0493425, C_{12}=-0.0234103, C_{16}=0.0135257, C_{20}=-0.00877315$, $C_{24}=0.00613892, C_{28}=-0.00453149, C_{32}=0.00348022, C_{36}=-0.00275574, C_{40}=0.00223562, \ldots$

Therefore, $b=a C_{0} / 2 \approx 1.1222 a$.

The maximum value of $C(\theta)$ is $\sqrt{2}$, which gives $\varepsilon=2 \sqrt{2} / C_{0}-1 \approx 0.260216$, and $|f(\theta)| \leq 1$, where

$$
f(\theta)=\sum_{m=1}^{\infty} f_{4 m} \cos (4 m \theta), \quad f_{4 m}=2 C_{4 m} /\left(\varepsilon C_{0}\right), \quad m \geq 1
$$

and

$f_{4}=-0.535742, \quad f_{8}=0.168973, \quad f_{12}=-0.0801686, f_{16}=0.0463187, \quad f_{20}=-0.0300436, f_{24}=0.0210227$, $f_{28}=-0.0155181, f_{32}=0.011918, f_{36}=-0.00943701, f_{40}=0.00765587, \ldots$

Note that with more terms retained in the Fourier series (92), the corresponding cross sectional shapes are closer to the square (Fig. 2). However the present method is for smooth cross sections of the vertical cylinder. It is impossible to obtain strains and deflections of ice plate by the present method for an exact square with sharp corners.

The effect of truncation in the series (92) for the shape function $f(\theta)$ with 3 and 6 terms is shown in Fig. 2. It is seen that even three terms in (92) well approximate a square cross section. The asymptotic expansion (91) up to order $O\left(\varepsilon^{3}\right)$ is used to calculate the amplitude of the normal strain component $|E(\theta)|$ as a function of the polar angle $\theta$. In numerical results, the series (92) is truncated with 3 terms and the series expansions of the potentials (70), (78) and (D.3) are truncated with 26 terms. It is observed that taking more than 26 terms in the series (70), (78) and (D.3) does not affect signifcantly the results for the strains. 
(a)

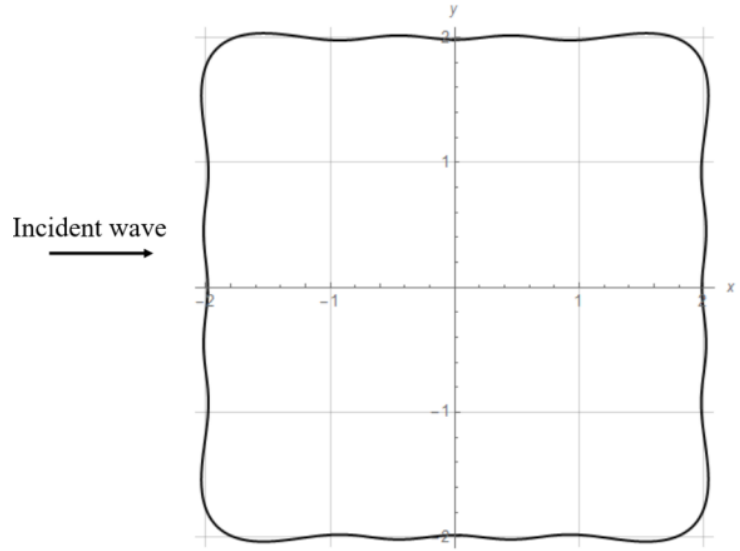

(b)

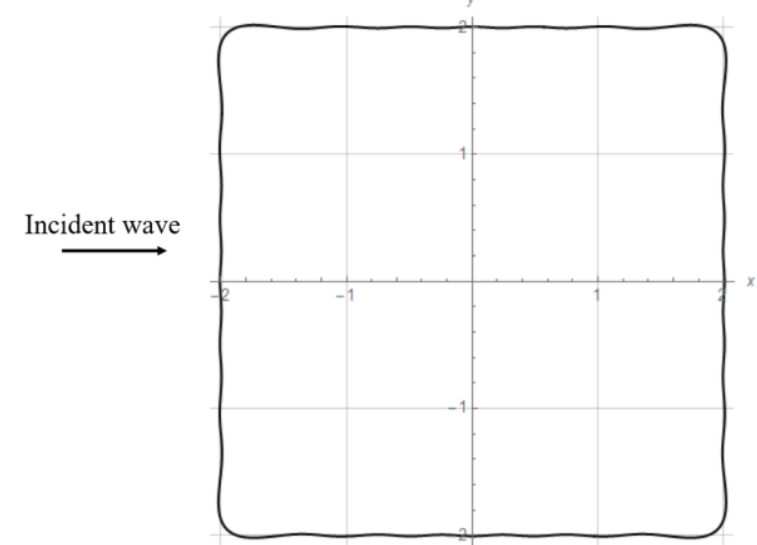

Fig. 2. Approximation of the square by the equation $r=b[1+\varepsilon f(\theta)]$ in the polar coordinates with (a) 3 terms and (b) 6 terms retained in Eq. (92).

For the cylinder with cross section close to a square of side length $4 \mathrm{~m}$ (Fig. 2(a)), the amplitude of the normal strain component, $|E(\theta)|$, as a function of the polar angle $\theta$ is shown in Fig. 3 for non-dimensional wave number $k H=0.7$ and in Fig. 4 for $k H=1$. In both figures, the results are compared for different ice thicknesses $h_{i}=0.2 \mathrm{~m}$ (solid line), $0.5 \mathrm{~m}$ (dotted line) and $1 \mathrm{~m}$ (dashed line). In both figures, the wave length is much greater than the dimensions of the cylinder. The wave length is about $135 \mathrm{~m}$ in Fig. 3 and $94 \mathrm{~m}$ in Fig. 4. The incident wave of amplitude $1 \mathrm{~cm}$ propagates from left to right, see Fig. 2.

(a)



(b)

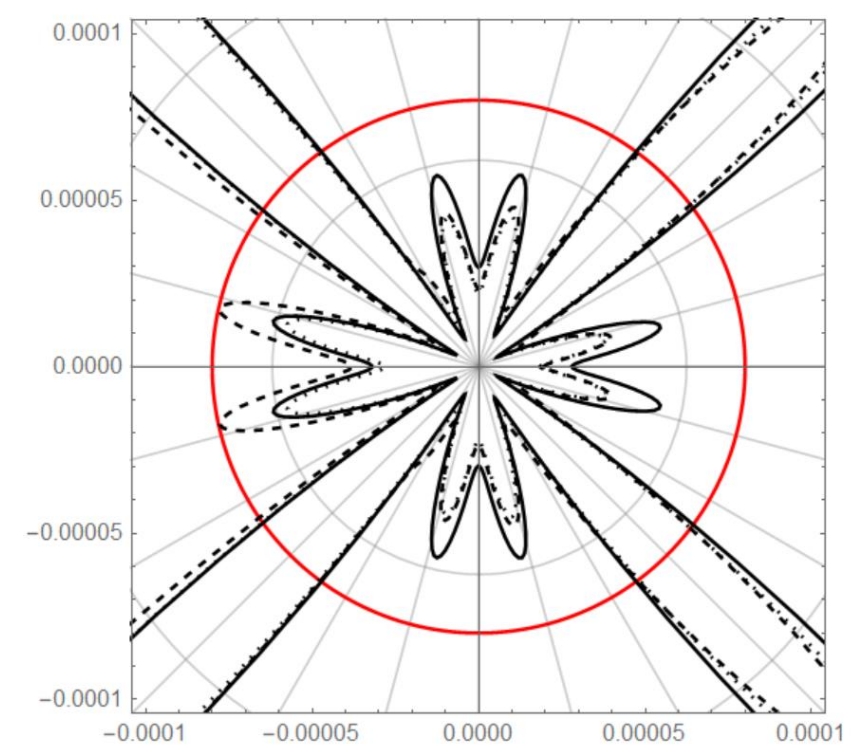

Fig. 3. The normal strain amplitudes at the contact line for a nearly square cylinder given in Fig. 2(a) for $A=0.01 \mathrm{~m}, H=15 \mathrm{~m}$, $k H=0.7, h_{i}=0.2 \mathrm{~m}$ (solid line), $h_{i}=0.5 \mathrm{~m}$ (dotted line), $h_{i}=1 \mathrm{~m}$ (dashed line). Yield strain $8 \times 10^{-5}$ is shown by a red line. The series for potentials and shape function (92) are truncated at 26 and 3 terms, respectively. Part (b) is the enlarged view of the central section of part (a). 
(a)

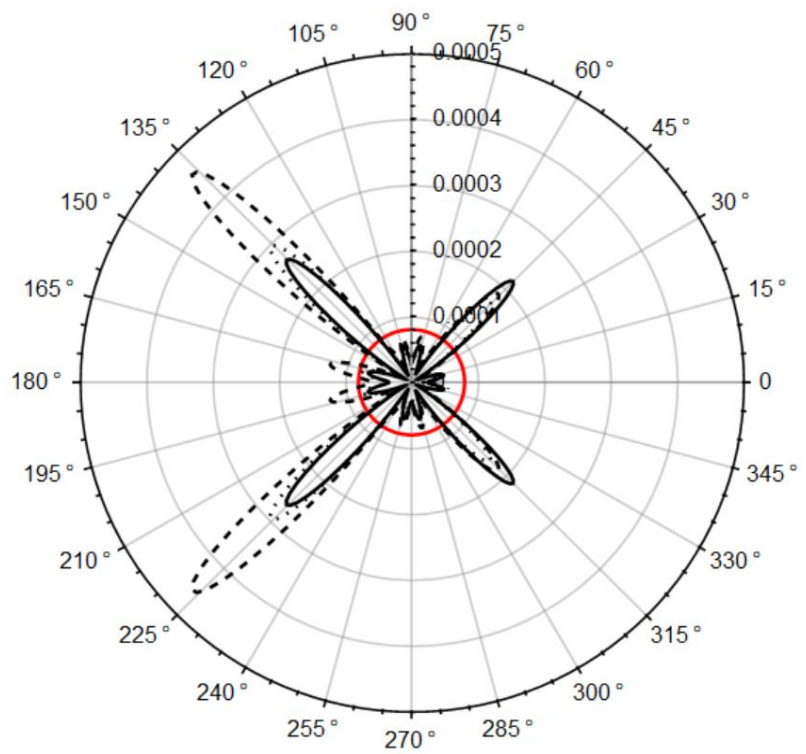

(b)

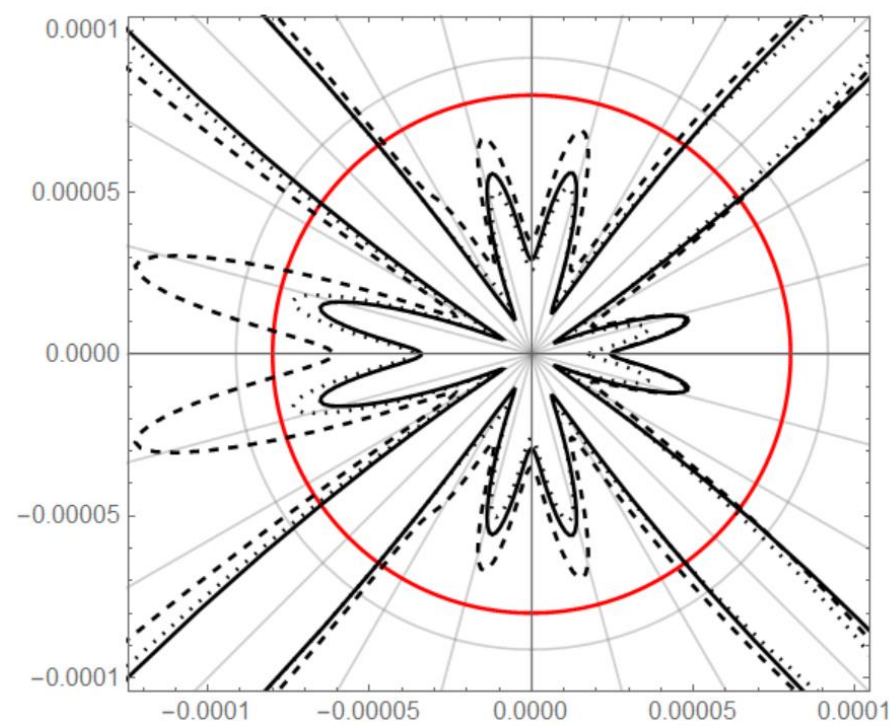

Fig. 4. The normal strain amplitudes at the contact line for a nearly square cylinder given in Fig. 2(a) for $A=0.01 \mathrm{~m}, H=15 \mathrm{~m}$, $k H=1, h_{i}=0.2 \mathrm{~m}$ (solid line), $h_{i}=0.5 \mathrm{~m}$ (dotted line), $h_{i}=1 \mathrm{~m}$ (dashed line). Yield strain $8 \times 10^{-5}$ is shown by a red line. The series for potentials and shape function (92) are truncated at 26 and 3 terms, respectively. Part (b) is the enlarged view of the central section of part (a).

In both figures 3 and 4 , the strain is concentrated at the corners of the square, so the breaking of ice at the corners is expected. For that reason the truncation of the shape function (92) is expected to affect the results. The effect of the number of terms retained in the shape function (92) on the results is demonstrated in Fig. 5 for the cross sections given in Fig 2 and for the ice thickness $h_{i}=0.2 \mathrm{~m}$, and $k H=1$. With more terms retained in the shape function, the normal strain amplitudes oscillate more than those obtained using fewer terms, see Fig. 5(b). This could be attributed to the number of oscillations in the cross sectional shapes in Fig. 2. There are more oscillations in Fig. 2(b) with six terms in the shape function than in Fig. 2(a) with three terms. The effect of the shape function on the strain distribution is great at the corners, see Fig. 5(a), with more terms retained in the shape function, the normal strain amplitudes are approximately $30 \%$ higher than those obtained with fewer terms.

The strain distribution depends on several factors; ice thickness, incident wave frequency and position of the corners of the cylinder with respect to the wave angle of attack. For $k H=0.7$ (Fig. 3), the thicker ice causes higher strains at the leading corners and smaller strains at the trailing corners. As for $k H=1$ (Fig. 4), a similar trend is observed at the leading corners but, with the thicker ice, the strains are higher than for $k H=0.7$ in Fig. 3. 
(a)

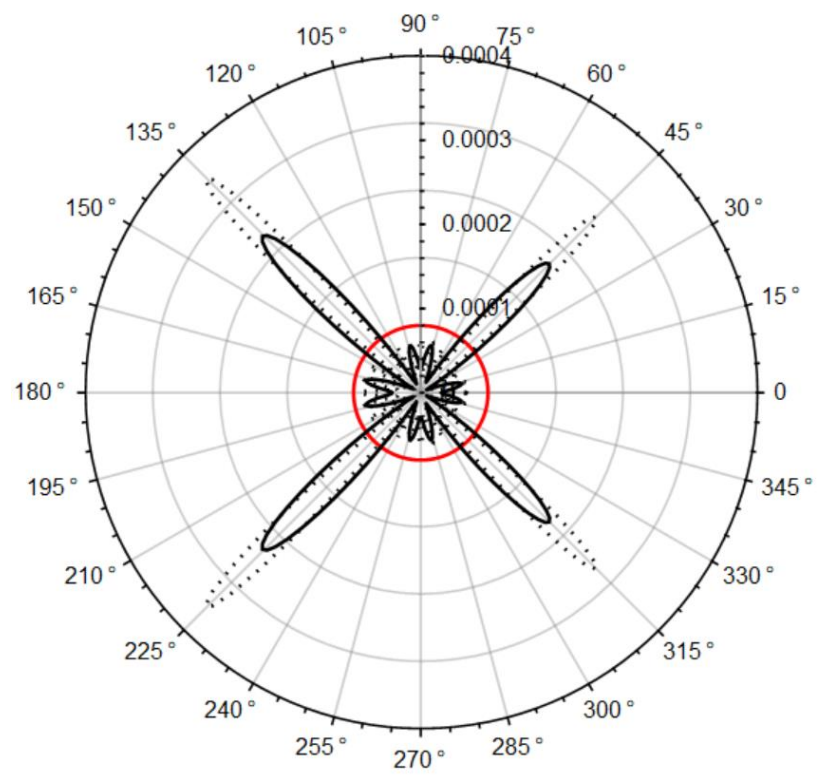

(b)

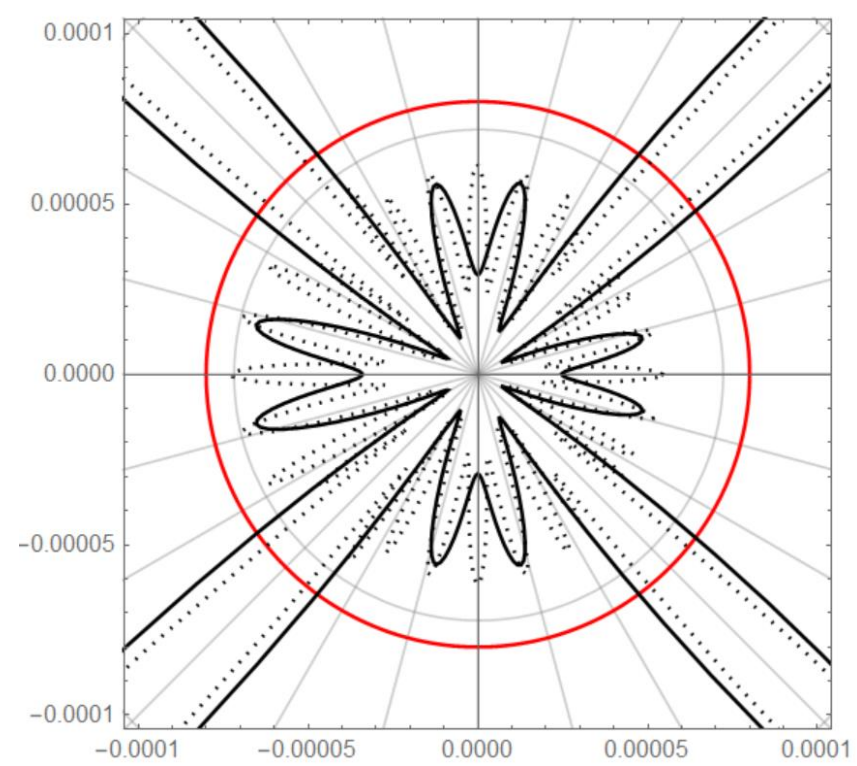

Fig. 5. The normal strain amplitudes at the contact line for a nearly square cylinder for $A=0.01 \mathrm{~m}, H=15 \mathrm{~m}, k H=1, h_{i}=0.2 \mathrm{~m}$. The series for potentials are truncated at 26 terms and shape function (92) is truncated at 3 terms (solid line), 6 terms (dotted line). Yield strain $8 \times 10^{-5}$ is shown by a red line. Part (b) is the enlarged view of the central section of part (a).

The effect of the number of the terms retained in the asymptotic expansion of the second normal derivative of the deflection (91) on the numerical results is demonstrated in Fig. 6. It is observed that with less terms retained in (91) the normal strain amplitudes at the corners are slightly overestimated than those with more terms.

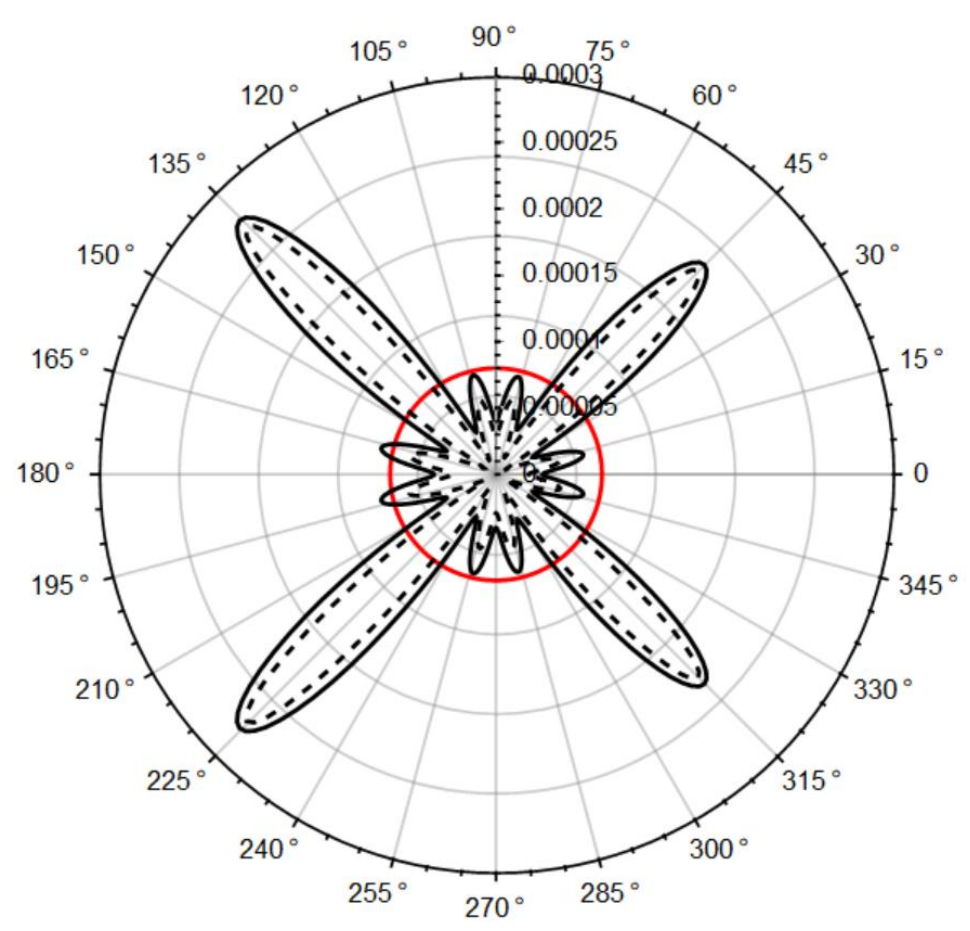

Fig. 6. The normal strain amplitudes calculated by the first-order approximation up to $O\left(\varepsilon^{2}\right)$ (solid line) and the second-order approximation up to $O\left(\varepsilon^{3}\right)$ (dashed line) in (91) at the contact line for a nearly square cylinder given in Fig. 2(a) for $A=0.01 \mathrm{~m}, H=15 \mathrm{~m}, k H=1, h_{i}=0.2 \mathrm{~m}$. Yield strain $8 \times 10^{-5}$ is shown by a red line. The series for potentials are truncated at 26 terms and the shape function (92) is truncated at 3 terms. 


\subsection{Strain Distribution on an Elliptic Cylinder}

In this section, a vertical cylinder with elliptic cross section is considered. The elliptic cross section is with eccentricity $e=\sqrt{1-\left(b_{1}^{2} / a_{1}^{2}\right)}=0.5$, where $a_{1}$ is the semi-major axis and $b_{1}$ is the semi-minor axis of the ellipse. The comparison of the exact shape of the ellipse with eccentricity $e=0.5$, semi-major axis $a_{1}=2$, and the approximation of the ellipse by the equation $r=b[1+\varepsilon f(\theta)]$ in the polar coordinates with two terms retained in the shape function $f(\theta)$ in (95), is shown in Fig. 7(a) . It is seen that the approximation of the ellipse with only two terms in the series (95) is reasonably good.

(a)

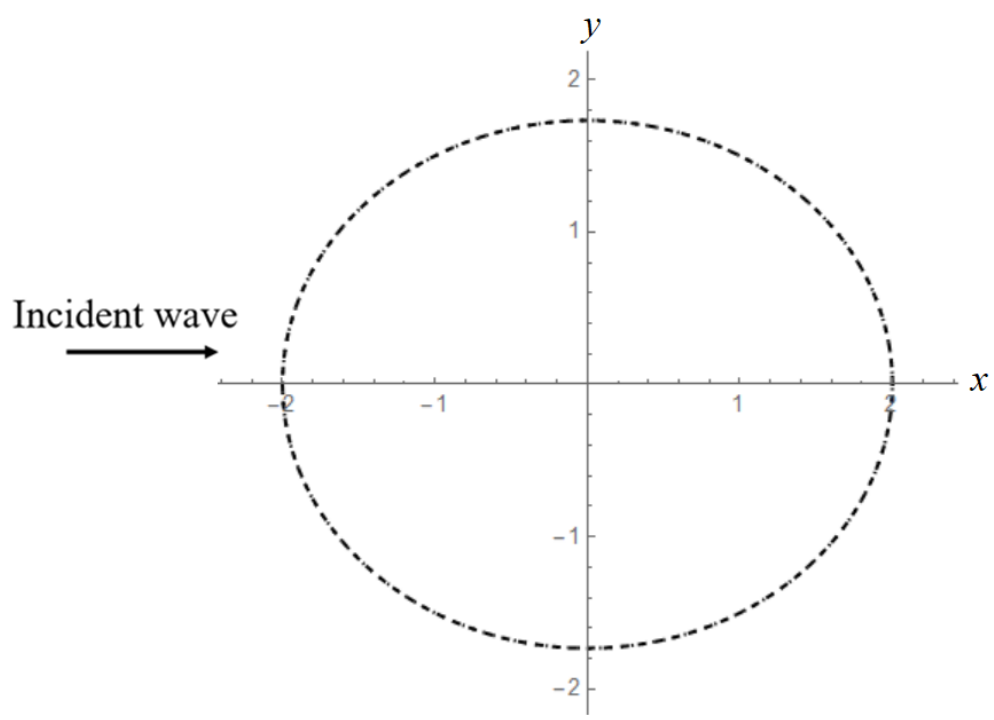

(b)

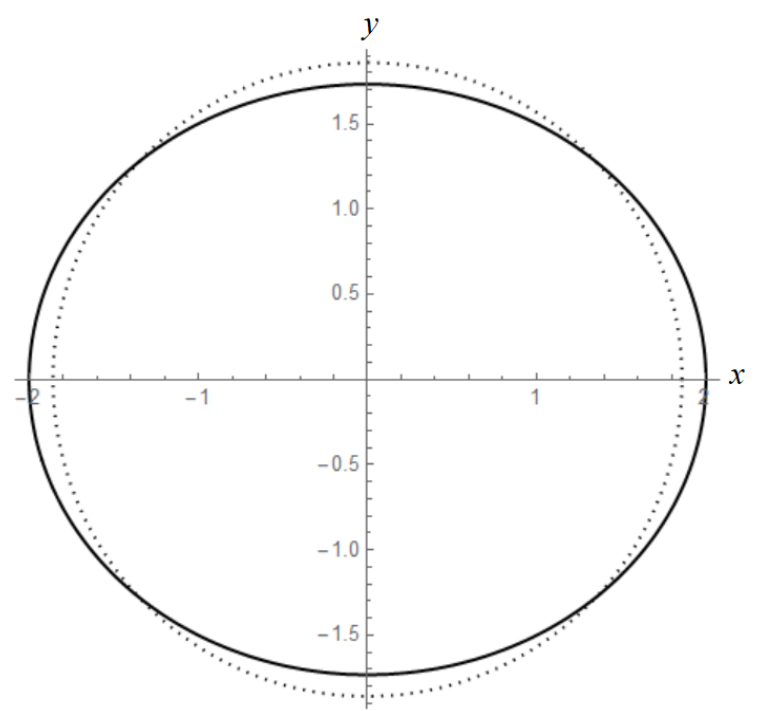

Fig. 7. (a) Ellipse with major axis $a_{1}=2 \mathrm{~m}$ and eccentricity $e=0.5$ (dashed line). Approximation of the ellipse by the equation $r=b[1+\varepsilon f(\theta)]$ with 2 terms retained in Eq. (95) (dotted line). (b) Approximation of the ellipse by the equation $r=b[1+\varepsilon f(\theta)]$ with 2 terms retained in Eq. (95) (solid line), circle with radius $b=0.9294 a_{1}$ (dotted line) where $a_{1}=2$ is the major axis of the ellipse.

Let equation $r=a_{1} C(\theta)$ describe the ellipse in the polar coordinates with the ellipse's centre at the origin, where

$$
C(\theta)=\frac{\sqrt{1-e^{2}}}{\sqrt{1-e^{2} \cos ^{2}(\theta)}}
$$

For numerical results, an elliptic cylinder whose cross section is given in Fig. 7(a) with $e=0.5$ is chosen. The Fourier coefficients of $C(\theta), 0 \leq \theta \leq 2 \pi$, in (93) are determined, and then the corresponding Fourier series is converted into the form $r=b[1+\varepsilon f(\theta)]$, identifying the values of $b, \varepsilon$, and the function $f(\theta)$. Then, the asymptotic formula (91) is used to calculate the amplitude of the normal strain component. 
An ellipse has two lines of symmetry, so the Fourier series of the function $C(\theta)$ contains $\cos (2 m \theta), m \geq 0$ terms only,

$$
C(\theta)=\frac{1}{2} C_{0}+\sum_{m=1}^{\infty} C_{2 m} \cos (2 m \theta)
$$

and for the ellipse with eccentricity $e=0.5$, some of the Fourier coefficients of $C(\theta)$ in (94) are given below,

$$
C_{0}=1.8588, C_{2}=0.0668, C_{4}=0.0036, C_{6}=0.0002, C_{8}=0.000014, C_{10}=8.7363 \times 10^{-7}, \ldots .
$$

Therefore, $\quad b=a_{1} C_{0} / 2 \approx 0.9294 a_{1}$. The maximum value of $C(\theta)$ is 1 , which gives $\varepsilon=2 / C_{0}-1 \approx 0.0759597$, and $|f(\theta)| \leq 1$, where

$$
f(\theta)=\sum_{m=1}^{\infty} f_{2 m} \cos (2 m \theta), \quad f_{2 m}=2 C_{2 m} /\left(\varepsilon C_{0}\right),
$$

and

$f_{2}=0.945807, \quad f_{4}=0.0509404, \quad f_{6}=0.003048, \quad f_{8}=0.0001915, \quad f_{10}=0.00001237$, $f_{12}=8.11669 \times 10^{-7}, \ldots$

For the elliptic cylinder with eccentricity $e=0.5$, the strain distribution around the cylinder has been calculated for incident wave amplitude $A=0.01 \mathrm{~m}$, ice thicknesses $h_{i}=0.2 \mathrm{~m}, 0.5 \mathrm{~m}$, and $1 \mathrm{~m}$, and for two different $k H$ values in Fig. 8 ( $k H=0.7$ in (a) and $k H=1$ in (b)). Third-order asymptotic expansion of (91) is used for numerical results and the series of the shape function (95) is truncated at two terms and the series expansion of the potentials (70), (78) and (D.3) are truncated at six terms. Increasing the terms in the series expansion of the potentials (70), (78) and (D.3) does not affect the results significantly.

It is observed that for the geometry and wave angle of attack considered in Fig. 7(a) the strains are higher at the leading side of the ellipse than at the trailing side and that for $k H=1$ the strains are higher than for $k H=0.7$ (Fig. 8). It is also observed that the greater the ice thickness, the greater the strains at the leading edge. The relation between the ice thickness and the strain is more complicated at the trailing edge with wave frequency having a considerable effect on the outcome. In both cases, the length of the incident wave is much longer than horizontal dimensions of the cylinder. 
(a)

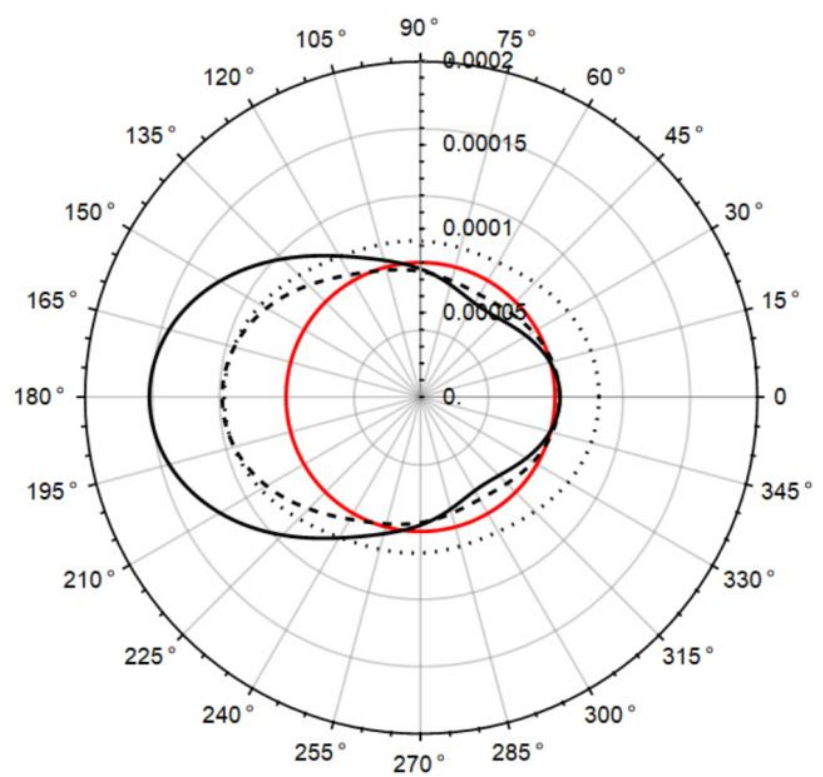

(b)

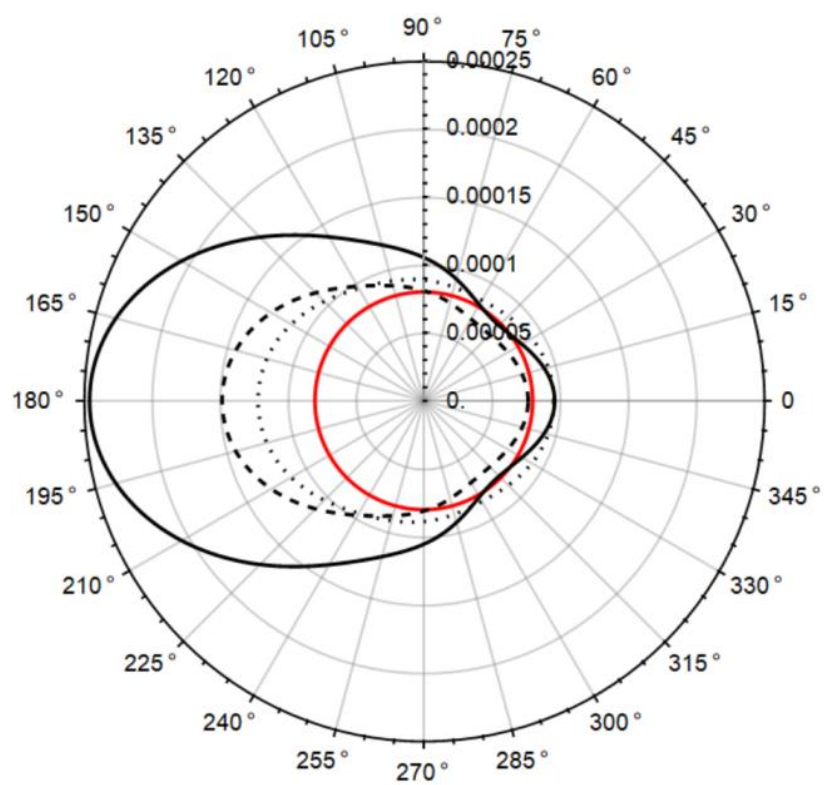

Fig. 8. The normal strain amplitudes at the contact line for an elliptic cylinder with eccentricity $e=0.5$, for $A=0.01 \mathrm{~m}$, $H=15 \mathrm{~m}$, (a) $k H=0.7$, (b) $k H=1$, and $h_{i}=0.2 \mathrm{~m}$ (dotted line), $h_{i}=0.5 \mathrm{~m}$ (dashed line), $h_{i}=1 \mathrm{~m}$ (solid line), and the series for potentials and shape function (95) are truncated at 6 and 2 terms, respectively. Yield strain $8 \times 10^{-5}$ is shown by a red line.

The effect of the number of terms retained in the asymptotic expansion (91) on the numerical results for the normal strain amplitudes at the contact line for an elliptic cylinder of Fig. 7(a) is demonstrated in Fig. 9. The normal strain amplitudes for $O\left(\varepsilon^{2}\right)$ (dashed line), $O\left(\varepsilon^{3}\right)$ (dotted line), $O\left(\varepsilon^{4}\right)$ (solid line) in (91) are compared and it is observed that the results are nearly the same.

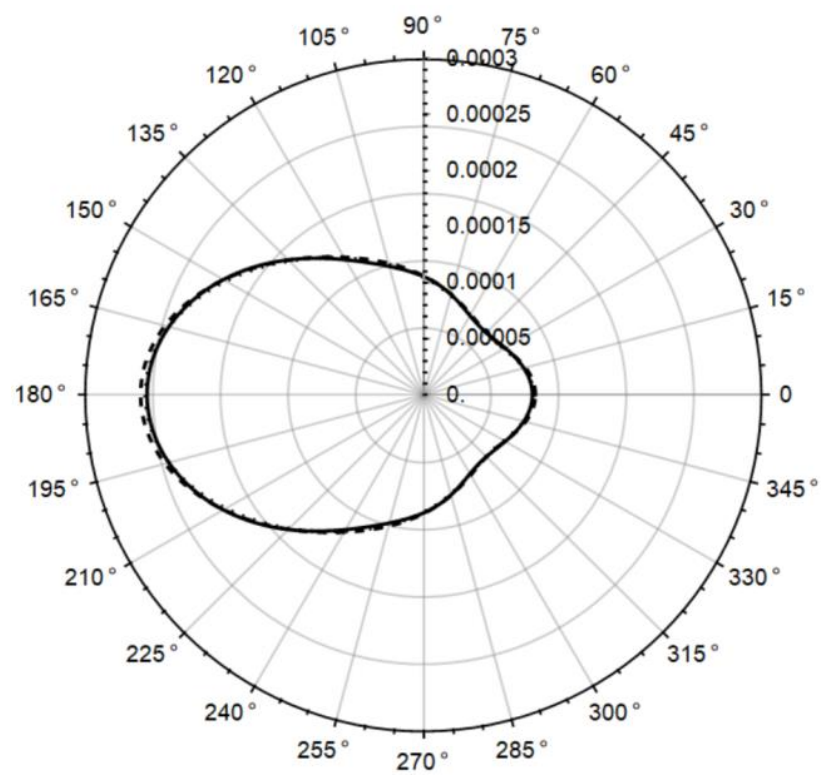

Fig. 9. The normal strain amplitudes up to order $O\left(\varepsilon^{2}\right)$ (dashed line), $O\left(\varepsilon^{3}\right)$ (dotted line), $O\left(\varepsilon^{4}\right)$ (solid line) in (91) at the contact line for elliptic cylinder given in Fig. 7(b) for $A=0.01 \mathrm{~m}, H=15 \mathrm{~m}, k H=1, h_{i}=1 \mathrm{~m}$. The series for potentials and shape function (95) are truncated at 6 and 2 terms, respectively. 
Figure 10 compares the normal strain amplitudes for a circular cylinder of radius $r=b \approx 0.9294 a_{1}$ and an elliptical cylinder $r=b[1+\varepsilon f(\theta)]$, see Fig. 7(b). It is observed that at the leading side the normal strain amplitude is higher for the elliptical cylinder than the one for the circular cylinder.

(a)

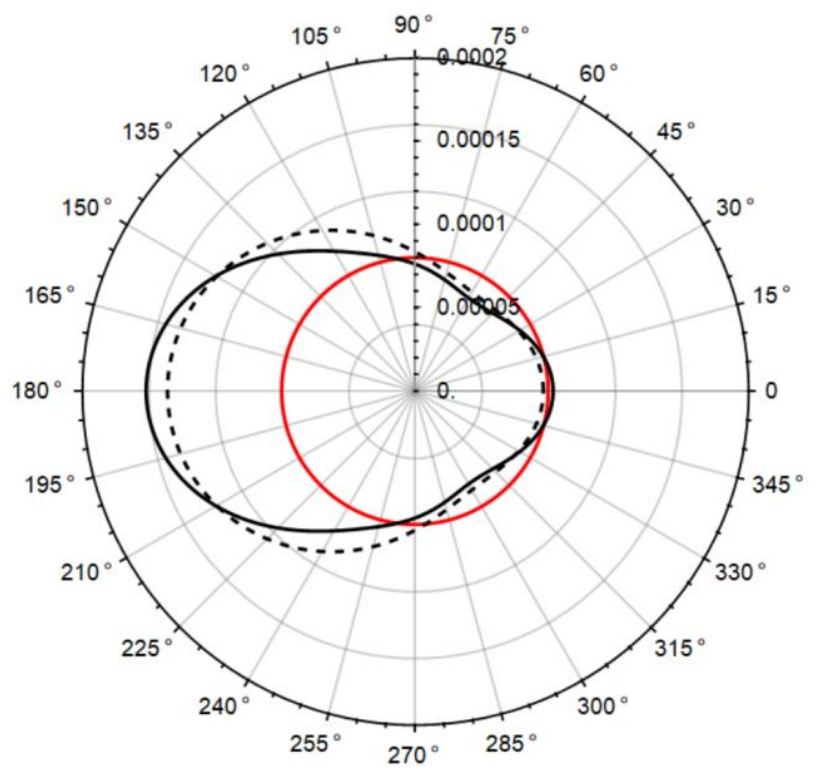

(b)

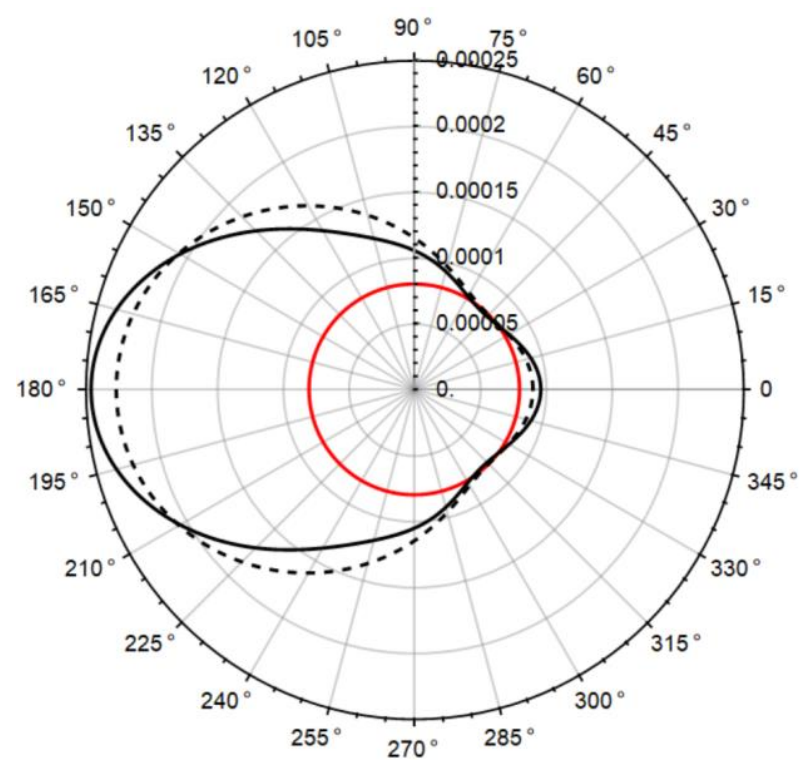

Fig. 10. The normal strain amplitudes at the contact line for an elliptic cylinder $r=b[1+\varepsilon f(\theta)]$ with eccentricity $e=0.5$ (solid line), for a circular cylinder of radius $b$ (dashed line) for $A=0.01 \mathrm{~m}, H=15 \mathrm{~m}, h_{i}=1 \mathrm{~m}$, (a) $k H=0.7$, (b) $k H=1$. Yield strain $8 \times 10^{-5}$ is shown by a red line.

\section{Conclusion}

The linear problem of diffraction of flexural-gravity waves from a vertical cylinder of smooth non-circular cross section has been investigated by the vertical mode method combined with an asymptotic method, under the assumption of clamped edge conditions. The third-order asymptotic solution of the problem has been obtained. The leading order term in the asymptotic expansion of the velocity potential is the solution of the diffraction problem for the circular cylinder. The higher order terms correspond to solutions of radiation problems for the circular cylinder. These problems differ only by the value of the normal derivative of the corresponding potential on the surface of the circular cylinder. The corresponding problems have been solved by the Fourier method. The numerical solution of the problem has been reduced to the operations with the Fourier coefficients of the potentials and the shape function. The numerical algorithm has been applied to the problems of hydro-elastic wave diffraction by an elliptical and a nearly square cylinder.

For the case of the nearly square cylinder, the strains are highest at the corners of the square, so the breaking of ice occurs at the corners as expected. Truncation of the shape function affects the results. The effect of the 
shape function on the strain distribution is greater at the corners. With more terms retained in the shape function, the normal strain amplitude is approximately $30 \%$ higher than with fewer terms.

For the case of elliptical cylinder, if it is aligned with the waves, the strain is higher at the leading side of the ellipse than the one at the trailing side. For shorter waves $(k H=1)$ the strains are higher than for longer waves $(k H=0.7)$. It is also observed that the greater the ice thickness, the greater the strain at the leading edge. The relation between the ice thickness and the strain is more complicated at the trailing edge with wave frequency having a considerable effect on the result.

An advantage of the present approach compared with the numerical solution of the problem by a boundaryelement method is that it provides the forces and the diffracted wave field in terms of the Fourier series which describes the deviation of the cylinder cross section from the circular one. Present method can be used to optimize the shape of a vertical cylinder in icy waters cylinder to break the ice.

In real applications, cylinders are always arranged in groups. Therefore, the analysis of the hydro-elastic interaction problem for several non-circular cylinders in ice would be helpful for further developments of the polar regions.

\section{Acknowledgements}

Preliminary results of this paper were reported at the conference "Days on Diffraction 2019" (Disibuyuk et al. [14]). This research was started while the first author was visiting the School of Mathematics, University of East Anglia, as a postdoctoral researcher in the period from February 2019 to February 2020. The visit and the study were supported by TÜBİTAK (Scientific and Technological Research Council of Turkey) grant (BIDEB-2219) and the TÜBİTAK project, (Grant No: 119N361) and this support is greatly acknowledged. 


\section{REFERENCES}

[1] C. Fox, V.A. Squire, On the oblique reflexion and transmission of ocean waves at shore fast sea ice, Philos. Trans. - R. Soc. London, A. (1994). https://doi.org/10.1098/rsta.1994.0044.

[2] J.B. Lawrie, I.D. Abrahams, An Orthogonality relation for a class of problems with high-order boundary conditions; Applications in sound-structure interaction, Q. J. Mech. Appl. Math. (1999). https://doi.org/10.1093/qjmam/52.2.161.

[3] T. Sahoo, T.L. Yip, A.T. Chwang, Scattering of surface waves by a semi-infinite floating elastic plate, Phys. Fluids. (2001). https://doi.org/10.1063/1.1408294.

[4] D. V. Evans, R. Porter, Wave scattering by narrow cracks in ice sheets floating on water of finite depth, J. Fluid Mech. (2003). https://doi.org/10.1017/S002211200300435X.

[5] P. Brocklehurst, A. Korobkin, E.I. Părău, Hydroelastic wave diffraction by a vertical cylinder, Philos. Trans. R. Soc. A Math. Phys. Eng. Sci. (2011). https://doi.org/10.1098/rsta.2011.0110.

[6] P. Brocklehurst, A.A. Korobkin, E.I. Părău, Interaction of hydro-elastic waves with a vertical wall, J. Eng. Math. (2010). https://doi.org/10.1007/s10665-010-9386-8.

[7] J. Bhattacharjee, C. Guedes Soares, Flexural gravity wave over a floating ice sheet near a vertical wall, J. Eng. Math. (2012). https://doi.org/10.1007/s10665-011-9511-3.

[8] A.A. Korobkin, S. Malenica, T. Khabakhpasheva, Interaction of flexural-gravity waves in ice cover with vertical walls, Philos. Trans. R. Soc. A Math. Phys. Eng. Sci. (2018). https://doi.org/10.1098/rsta.2017.0347.

[9] A.A. Korobkin, S. Malenica, T. Khabakhpasheva, The vertical mode method in the problems of flexural-gravity waves diffracted by a vertical cylinder, Appl. Ocean Res. (2019). https://doi.org/10.1016/j.apor.2018.11.012.

[10] K. Ren, G.X. Wu, C.Y. Ji, Diffraction of hydroelastic waves by multiple vertical circular cylinders, J. Eng. Math. (2018). https://doi.org/10.1007/s10665-018-9973-7.

[11] N.B. Dişibüyük, A.A. Korobkin, O. Yilmaz, Linear wave interaction with a vertical cylinder of arbitrary cross section: An asymptotic approach, J. Waterw. Port, Coast. Ocean Eng. (2017). https://doi.org/10.1061/(ASCE)WW.1943-5460.0000407.

[12] S. Timoshenko, S. Woinowsky-Krieger, Theory of plates and shells, 2nd ed., McGraw-Hill Book Co., Inc., 1959.

[13] D.E. Timokhov, L. A., Heisin, Dynamics of sea ice, Gidrometeoizdat. (1987) Leningrad 272 (in Russian).

[14] N.B. Disibuyuk, A.A. Korobkin, O. Y1lmaz, Diffraction of flexural-gravity waves from a vertical cylinder of non-circular cross section, in: Days Diffr., St. Petersburg, Russia, 2019.

[15] R.C. MacCamy, R.A. Fuchs, Wave Forces on Piles: A Diffraction Theory, U.S. Army Coast. Eng. 
Res. Cent. (Formerly Beach Eros. Board), Tech. Memo. No. 69. (1954).

[16] G.M. Fichtenholtz, Course of differential and integral calculus, Fizmatlit, Moscow, 2001.

\section{APPENDIX A}

\section{Diffraction Problem by a Cylinder of Arbitrary Cross Section without Edge Conditions}

The solution of the problem (18) - (20) is outlined here. The detailed solution can be found in [11]. Some operators on the surface of the cylinder are introduced in this Appendix. They are used in the main part of the paper, see equations (54) - (56).

The body boundary condition (19) reads in the polar coordinates,

$$
\frac{\partial \Phi}{\partial r}(b[1+\varepsilon f(\theta)], \theta)-\frac{\varepsilon f^{\prime}(\theta)}{b[1+\varepsilon f(\theta)]^{2}} \frac{\partial \Phi}{\partial \theta}(b[1+\varepsilon f(\theta)], \theta)=0 .
$$

The derivatives $\partial \Phi / \partial r$ and $\partial \Phi / \partial \theta$ on the surface of the cylinder, $r=b[1+\varepsilon f(\theta)]$, in the boundary condition (A.1) are approximated by their Taylor series in $\varepsilon$ up to $O\left(\varepsilon^{4}\right)$ as $\varepsilon \rightarrow 0$. These series together the third-order asymptotic expansion of the potential $\Phi(r, \theta)$,

$$
\Phi(r, \theta)=\Phi_{0}(r, \theta)+\varepsilon \Phi_{1}(r, \theta)+\varepsilon^{2} \Phi_{2}(r, \theta)+\varepsilon^{3} \Phi_{3}(r, \theta)+O\left(\varepsilon^{4}\right),
$$

are substituted in the boundary condition (A.1). At each asymptotic order the following boundary conditions are obtained at $r=b$,

$$
\begin{gathered}
\Phi_{0, r}(b, \theta)=0, \\
\Phi_{j, r}(b, \theta)=G_{j}(\theta), \quad j=1,2,3,
\end{gathered}
$$

where

$$
\begin{gathered}
G_{1}(\theta)=L_{10}\left[\Phi_{0}(b, \theta)\right], \\
G_{2}(\theta)=L_{10}\left[\Phi_{1}(b, \theta)\right]+L_{20}\left[\Phi_{0}(b, \theta)\right], \\
G_{3}(\theta)=L_{10}\left[\Phi_{2}(b, \theta)\right]+L_{20}\left[\Phi_{1}(b, \theta)\right]+L_{30}\left[\Phi_{0}(b, \theta)\right] .
\end{gathered}
$$

The differential operators, $L_{j n}, j=1,2,3, n=-2,-1,0, \ldots$, are given as

$L_{1 n}=\frac{1}{b}\left[f(\theta) \frac{\partial^{2}}{\partial \theta^{2}}+f^{\prime}(\theta) \frac{\partial}{\partial \theta}+\kappa_{n}^{2} \frac{b^{2}}{H^{2}} f(\theta)\right]$, 


$$
\begin{aligned}
& L_{2 n}=\frac{1}{2 b}\left[-f^{2}(\theta) \frac{\partial^{2}}{\partial \theta^{2}}-2 f(\theta) f^{\prime}(\theta) \frac{\partial}{\partial \theta}+\kappa_{n}^{2} \frac{b^{2}}{H^{2}} f^{2}(\theta)\right], \\
& L_{3 n}=\frac{1}{3 b}\left[\begin{array}{rl}
f^{3}(\theta) & \frac{\partial^{4}}{\partial \theta^{4}}+6 f^{2}(\theta) f^{\prime}(\theta) \frac{\partial^{3}}{\partial \theta^{3}} \\
+ & {\left[f^{3}(\theta)+2 f^{3}(\theta) \kappa_{n}^{2} \frac{b^{2}}{H^{2}}+6 f(\theta)\left[f^{\prime}(\theta)\right]^{2}+\frac{9 f^{2}(\theta) f^{\prime \prime}(\theta)}{2}\right] \frac{\partial^{2}}{\partial \theta^{2}}} \\
+ & \left.\left[3 f^{2}(\theta) f^{\prime}(\theta)+6 f^{2}(\theta) f^{\prime}(\theta) \kappa_{n}^{2} \frac{b^{2}}{H^{2}}+\frac{3 f^{2}(\theta) f^{\prime \prime \prime}(\theta)}{2}+3 f(\theta) f^{\prime}(\theta) f^{\prime \prime}(\theta)\right] \frac{\partial}{\partial \theta}\right], \\
+ & f^{3}(\theta) \kappa_{n}^{4} \frac{b^{4}}{H^{4}}+3 f(\theta)\left[f^{\prime}(\theta)\right]^{2} \kappa_{n}^{2} \frac{b^{2}}{H^{2}}+\frac{3 f^{2}(\theta) f^{\prime \prime}(\theta)}{2} \kappa_{n}^{2} \frac{b^{2}}{H^{2}},
\end{array}\right]
\end{aligned}
$$

where $\kappa_{n}, n=-2,-1,0, \ldots$ are roots of the dispersion relation (12). The differential operators, $L_{j 0}, j=1,2,3$, are used in the solution of the wave diffraction problem without evanescent waves $(n=0)$. The operators $L_{j n}$ with $n \neq 0$ correspond to evanescent waves. They are used in equations (54) - (56) of the main part of the paper. At the leading order, the boundary condition (A.3) provides the solution for the circular cylinder, $r=b$, given by MacCamy and Fuchs [15],

$$
\Phi_{0}(r, \theta)=\sum_{m=0}^{\infty} \epsilon_{m} i^{m}\left[J_{m}(k r)-\frac{J_{m}^{\prime}(k b)}{H_{m}^{(1) '}(k b)} H_{m}^{(1)}(k r)\right] \cos (m \theta)
$$

where $\epsilon_{m}$ is the Neumann symbol, $\epsilon_{0}=1, \epsilon_{m}=2$ for $m \geq 1, J_{m}(r)$ is the Bessel function of the first kind with order $m, H_{m}^{(1)}(r)$ is the Hankel function of the first kind with order $m$ corresponding to outwardpropagating cylindrical waves. By using the Wronskian identity, $J_{m}(r) H_{m}^{(1) '}(r)-J_{m}{ }^{\prime}(r) H_{m}^{(1)}(r)=2 i /(\pi r)$, the potential $\Phi_{0}(r, \theta)$ on the surface of the circular cylinder, $r=b$, is given by

$$
\Phi_{0}(b, \theta)=\frac{2 i}{\pi k b} \sum_{m=0}^{\infty} \frac{\epsilon_{m} i^{m}}{H_{m}^{(1)^{\prime}}(k b)} \cos (m \theta) .
$$

At the following orders as $\varepsilon \rightarrow 0$, the functions $G_{n}(\theta), n=1,2,3$ in (A.5) - (A.7) are the sums of the products of the functions $f(\theta), f^{\prime}(\theta), f^{\prime \prime}(\theta), \Phi_{0}(b, \theta), \Phi_{1}(b, \theta), \Phi_{2}(b, \theta)$ and derivatives of the potentials $\Phi_{0}(r, \theta), \Phi_{1}(r, \theta), \Phi_{2}(r, \theta)$ in $\theta$. Starting from the solution (A.12) for the circular cylinder and a given function $f(\theta)$, the right-hand side $G_{1}(\theta)$ in (A.4) is calculated and then the outwardpropagating wave solution, $\Phi_{1}(r, \theta)$, of equation (18) subject to the boundary condition (A.4) is determined. By using the obtained potential $\Phi_{1}(r, \theta)$, we calculate $G_{2}(\theta)$ and determine $\Phi_{2}(r, \theta)$, and so 
on. The boundary value problems for the potentials $\Phi_{n}(r, \theta), n=1,2,3$ are similar to each other, they differ only by the functions $G_{n}(\theta)$. By using the Fourier series of the functions $G_{n}(\theta)$,

$$
G_{n}(\theta)=\sum_{m=0}^{\infty} \frac{\epsilon_{m}}{2}\left[G_{n m}^{(\mathrm{c})} \cos (m \theta)+G_{n m}^{(\mathrm{s})} \sin (m \theta)\right]
$$

the potentials are given by

$$
\Phi_{n}(r, \theta)=\sum_{m=0}^{\infty} \frac{\epsilon_{m}}{2}\left[G_{n m}^{(\mathrm{c})} \cos (m \theta)+G_{n m}^{(\mathrm{s})} \sin (m \theta)\right] \frac{H_{m}^{(1)}(k r)}{k H_{m}^{(1)^{\prime}}(k b)}, \quad n=1,2,3 .
$$

Note that, $\Phi_{n}(r, \theta)$ and their derivatives are obtained in the form of their Fourier series. Calculations of the functions $G_{n}(\theta)$ and their Fourier coefficients are reduced to multiplication and summation of Fourier series (see Appendix B). If the coefficients in the Fourier series of the shape function $f(\theta)$ are known,

$$
f(\theta)=\sum_{m=0}^{\infty} \frac{\epsilon_{m}}{2}\left[f_{m}^{(\mathrm{c})} \cos (m \theta)+f_{m}^{(\mathrm{s})} \sin (m \theta)\right]
$$

then the derivatives $f^{\prime}(\theta), \Phi_{0, \theta}(b, \theta)$ and $\Phi_{0, \theta \theta}(b, \theta)$ are calculated by differentiating (A.12) and (A.15) term by term. Then the Fourier coefficients $G_{1 m}^{(\mathrm{c})}$ and $G_{1 m}^{(s)}$ of the right-hand side in (A.4) for $j=1$ can be determined. Finally, the solution $\Phi_{1}(r, \theta)$ is given by (A.14). Similar arguments are applied to the higherorder problems for $\Phi_{2}(r, \theta)$ and $\Phi_{3}(r, \theta)$. It is seen that the asymptotic solution (A.2) of the problem is obtained by operating with the Fourier coefficients of the potentials $\Phi_{n}(b, \theta)$ and the Fourier coefficients of the function $f(\theta)$, which describes the cross-sectional shape of the vertical cylinder.

\section{APPENDIX B}

\section{Multiplication of Fourier Series}

The multiplication of two Fourier series,

$$
\begin{gathered}
g(\theta) \sim \frac{a_{0}}{2}+\sum_{m=1}^{\infty} a_{m} \cos (m \theta)+b_{m} \sin (m \theta), \\
h(\theta) \sim \frac{\alpha_{0}}{2}+\sum_{m=1}^{\infty} \alpha_{m} \cos (m \theta)+\beta_{m} \sin (m \theta),
\end{gathered}
$$

provides the Fourier series 


$$
g(\theta) h(\theta) \sim \frac{A_{0}}{2}+\sum_{m=1}^{\infty} A_{m} \cos (m \theta)+B_{m} \sin (m \theta)
$$

where, see [16],

$$
\begin{aligned}
& A_{n}=\frac{a_{0} \alpha_{n}}{2}+\frac{1}{2} \sum_{m=1}^{\infty}\left[a_{m}\left(\alpha_{m+n}+\alpha_{m-n}\right)+b_{m}\left(\beta_{m+n}+\beta_{m-n}\right)\right], \\
& B_{n}=\frac{a_{0} \beta_{n}}{2}+\frac{1}{2} \sum_{m=1}^{\infty}\left[a_{m}\left(\beta_{m+n}-\beta_{m-n}\right)-b_{m}\left(\alpha_{m+n}-\alpha_{m-n}\right)\right],
\end{aligned}
$$

$\beta_{m-n}=-\beta_{n-m}$ and $\alpha_{m-n}=\alpha_{n-m}$ if $m-n<0$.

\section{APPENDIX C}

\section{Derivation of Boundary Condition (42)}

Following Section 3 in [8], the boundary condition (42) is derived on the surface of the cylinder, $r=b[1+\varepsilon f(\theta)]$. We use orthogonality of the vertical modes (36) in terms of the product (37), and the series (39) to evaluate the following product

$$
\left\langle\frac{\partial \varphi}{\partial n}, f_{k}(\hat{z})\right\rangle=\left\langle\sum_{s=-2}^{\infty} \frac{\partial \varphi_{s}}{\partial n} f_{s}(\hat{z}), f_{k}(\hat{z})\right\rangle=\sum_{s=-2}^{\infty} \frac{\partial \varphi_{s}}{\partial n}\left\langle f_{s}(\hat{z}), f_{k}(\hat{z})\right\rangle=\frac{\partial \varphi_{k}}{\partial n} Q_{k},
$$

where $r>b[1+\varepsilon f(\theta)], k \geq-2$, and $Q_{k}$ are given by (38).

Next the same product is calculated by definition (37), where $r>b[1+\varepsilon f(\theta)]$,

$$
\left\langle\frac{\partial \varphi}{\partial n}, f_{k}(\hat{z})\right\rangle=\int_{-1}^{0} \frac{\partial \varphi}{\partial n} f_{k}(\hat{z}) \mathrm{d} \hat{z}+\left.\frac{1}{q} \frac{\partial^{3}}{\partial \hat{z}^{3}}\left(\frac{\partial \varphi}{\partial n}\right)\right|_{\hat{z}=0} f_{k}^{\prime}(0)+\left.\frac{1}{q} \frac{\partial}{\partial \hat{z}}\left(\frac{\partial \varphi}{\partial n}\right)\right|_{\hat{z}=0} f_{k}^{\prime \prime \prime}(0) .
$$

The results in (C.1) and (C.2) should be equal to each other even in the limit $r \rightarrow b[1+\varepsilon f(\theta)]$, where we approach the surface of the cylinder. In this limit, the integral in (C.2) tends to zero because of (29), and the last term also tends to zero because the kinematic condition (26) and the edge condition (23). Hence, the only contribution from the right-hand side of (C.2) comes from the second term,

$$
\lim _{r \rightarrow b[1+\varepsilon f(\theta)]} \frac{1}{q}\left[\left.\frac{\partial^{3}}{\partial \hat{z}^{3}}\left(\frac{\partial \varphi}{\partial n}\right)\right|_{\hat{z}=0} f_{k}^{\prime}(0)\right]=\frac{H^{3}}{q} \lim _{r \rightarrow b[1+\varepsilon f(\theta)]}\left[\left.\frac{\partial^{3}}{\partial z^{3}}\left(\frac{\partial \varphi}{\partial n}\right)\right|_{z=0}\right]=\frac{H^{3}}{q} \lim _{r \rightarrow b[1+\varepsilon f(\theta)]}\left[\left.\frac{\partial}{\partial n}\left(\frac{\partial^{3} \varphi}{\partial z^{3}}\right)\right|_{z=0}\right] .
$$

Taking the derivative of the Laplace's equation (27) with respect to $z$ and then using the condition (26) gives

$$
\left.\left(\frac{\partial^{3} \varphi}{\partial z^{3}}\right)\right|_{z=0}=-\nabla^{2} W(r, \theta)
$$

Combining (C.3) and (C.4) we obtain 


$$
\lim _{r \rightarrow b[1+\varepsilon f(\theta)]} \frac{1}{q}\left[\left.\frac{\partial^{3}}{\partial \hat{z}^{3}}\left(\frac{\partial \varphi}{\partial n}\right)\right|_{\hat{z}=0} f_{k}^{\prime}(0)\right]=-\frac{H^{3}}{q} \lim _{r \rightarrow b[1+\varepsilon f(\theta)]} \frac{\partial}{\partial n}\left[\nabla^{2} W(r, \theta)\right]=-\left.\frac{H^{3}}{q} \frac{\partial}{\partial n}\left[\nabla^{2} W\right]\right|_{r=b[1+\varepsilon f(\theta)]} .
$$

Equating (C.1) and (C.2) in the limit, we find,

$$
\lim _{r \rightarrow b[1+\varepsilon f(\theta)]}\left\langle\frac{\partial \varphi}{\partial n}, f_{k}(\hat{z})\right\rangle=\frac{\partial \varphi_{k}}{\partial n} Q_{k}=-\left.\frac{H^{3}}{q} \frac{\partial}{\partial n}\left[\nabla^{2} W\right]\right|_{r=b[1+\varepsilon f(\theta)]},
$$

which leads to the boundary condition (42),

$$
\frac{\partial \varphi_{k}}{\partial n}=-\frac{H^{3}}{q Q_{k}} \alpha(\theta, \varepsilon), \quad \alpha(\theta, \varepsilon)=\left.\frac{\partial}{\partial n}\left[\nabla^{2} W\right]\right|_{r=b[1+\varepsilon f(\theta)]} \quad(r=b[1+\varepsilon f(\theta)]),
$$

where $k \geq-2$. The function $\alpha(\theta, \varepsilon)$ provides the shear force along the contact line. It is unknown in advance and should be determined by using the conditions at the contact line. Note that, this function does not depend on the index of the vertical mode $k$.

\section{APPENDIX D}

Determination of the potentials $\tilde{\varphi}_{n j}(r, \theta)$

Here, the potentials $\tilde{\varphi}_{n j}(r, \theta), j=2,3, n=-2,-1,0,1, \ldots$, in (48) and the unknown functions $\alpha_{j}(\theta)$, $j=2,3$, in (49) are determined. Second-order potentials, $\tilde{\varphi}_{n 2}(r, \theta), n=-2,-1,0,1, \ldots$, satisfy the following boundary value problems,

$$
\begin{gathered}
\nabla^{2} \tilde{\varphi}_{n 2}+\frac{\kappa_{n}^{2}}{H^{2}} \tilde{\varphi}_{n 2}=0 \quad(r>b), \\
\tilde{\varphi}_{n 2, r}(b, \theta)=\tilde{G}_{n 2}(\theta)+\frac{1}{2}\left[f^{\prime}(\theta)\right]^{2} \alpha_{0}(\theta)+\alpha_{2}(\theta), \\
\lim _{r \rightarrow \infty} r^{1 / 2}\left[\frac{\partial \tilde{\varphi}_{n 2}}{\partial r}-i \frac{\kappa_{n}}{H} \tilde{\varphi}_{n 2}\right]=0 .
\end{gathered}
$$

The second-order correction deflection $W_{2}(r, \theta)$ is given by

$$
W_{2}(r, \theta)=-\frac{H^{2}}{q} \sum_{n=-2}^{\infty} \frac{\tilde{\varphi}_{n 2}(r, \theta)}{Q_{n}} .
$$

The deflection (D.2) should satisfy the edge condition (64). 
Let the second term on the right-hand side of the boundary condition in (D.1) at $r=b$ be presented by its Fourier series,

$$
\gamma_{1}(\theta)=\frac{1}{2}\left[f^{\prime}(\theta)\right]^{2} \alpha_{0}(\theta)=\sum_{m=0}^{\infty} \frac{\epsilon_{m}}{2}\left[\gamma_{1 m}^{(c)} \cos (m \theta)+\gamma_{1 m}^{(s)} \sin (m \theta)\right]
$$

Then the boundary condition in (D.1),

$$
\tilde{\varphi}_{n 2, r}(b, \theta)=\tilde{G}_{n 2}(\theta)+\gamma_{1}(\theta)+\alpha_{2}(\theta),
$$

provides, the second-order potentials, $\tilde{\varphi}_{n 2}(r, \theta), n=-2,-1,0,1, \ldots$, as

$$
\tilde{\varphi}_{n 2}(r, \theta)=\sum_{m=0}^{\infty} \frac{\epsilon_{m}}{2}\left\{\left[\tilde{G}_{n 2 m}^{(c)}+\gamma_{1 m}^{(c)}+\alpha_{2 m}^{(c)}\right] \cos (m \theta)+\left[\tilde{G}_{n 2 m}^{(s)}+\gamma_{1 m}^{(s)}+\alpha_{2 m}^{(s)}\right] \sin (m \theta)\right\} R_{n m}(r), \quad n \geq-2 .
$$

The deflection (D.2) and the edge condition (64) give

$$
\begin{aligned}
-\frac{H^{2}}{q} \sum_{n=-2}^{\infty} \frac{\tilde{\varphi}_{n 2}(b, \theta)}{Q_{n}}= & {\left[\Phi_{2}(b, \theta)+b f(\theta) \Phi_{1, r}(b, \theta)+\frac{[b f(\theta)]^{2}}{2} \Phi_{0, r r}(b, \theta)\right] } \\
& -b f(\theta)\left[-\frac{H^{2}}{q} \sum_{n=-2}^{\infty} \frac{\tilde{\varphi}_{n 1, r}(b, \theta)}{Q_{n}}\right]-\frac{[b f(\theta)]^{2}}{2}\left[-\frac{H^{2}}{q} \sum_{n=-2}^{\infty} \frac{\tilde{\varphi}_{n 0, r r}(b, \theta)}{Q_{n}}\right] .
\end{aligned}
$$

The right-hand side of (D.4) consists of summation and multiplication of Fourier series which together provide a Fourier series (see Appendix B),

$$
s_{1}(\theta)=\sum_{m=0}^{\infty} \frac{\epsilon_{m}}{2}\left[s_{1 m}^{(c)} \cos (m \theta)+s_{1 m}^{(s)} \sin (m \theta)\right]
$$

Now (D.4) can be written as follows:

$$
\begin{aligned}
-\frac{H^{2}}{q} \sum_{m=0}^{\infty} \frac{\epsilon_{m}}{2}\left\{\left(\left[\gamma_{1 m}^{(c)}+\alpha_{2 m}^{(c)}\right] g_{m}^{-1}+\tilde{g}_{2 m}^{(c)}\right) \cos (m \theta)+\left(\left[\gamma_{1 m}^{(s)}+\alpha_{2 m}^{(s)}\right]\right.\right. & \left.\left.g_{m}^{-1}+\tilde{g}_{2 m}^{(s)}\right) \sin (m \theta)\right\} \\
& =\sum_{m=0}^{\infty} \frac{\epsilon_{m}}{2}\left[s_{1 m}^{(c)} \cos (m \theta)+s_{1 m}^{(s)} \sin (m \theta)\right]
\end{aligned}
$$

where $\tilde{g}_{2 m}^{(c)}$ and $\tilde{g}_{2 m}^{(s)}$ are defined in (79). Comparing the coefficients in these Fourier series, we find

$$
\begin{gathered}
\alpha_{2 m}^{(c)}=\left(-\frac{q}{H^{2}} s_{1 m}^{(c)}-\tilde{g}_{2 m}^{(c)}\right) g_{m}-\gamma_{1 m}^{(c)}, \quad m=0,1,2,3, \ldots, \\
\alpha_{2 m}^{(s)}=\left(-\frac{q}{H^{2}} s_{1 m}^{(s)}-\tilde{g}_{2 m}^{(s)}\right) g_{m}-\gamma_{1 m}^{(s)}, \quad m=1,2,3, \ldots
\end{gathered}
$$

A similar procedure is applied to find the unknown Fourier coefficients of $\alpha_{3}(\theta)$ using condition (65). The third-order potentials, $\tilde{\varphi}_{n 3}(r, \theta), n=-2,-1,0,1, \ldots$, satisfy the following boundary value problem,

$$
\nabla^{2} \tilde{\varphi}_{n 3}+\frac{\kappa_{n}^{2}}{H^{2}} \tilde{\varphi}_{n 3}=0 \quad(r>b)
$$




$$
\begin{gathered}
\tilde{\varphi}_{n 3, r}(b, \theta)=\tilde{G}_{n 3}(\theta)-f(\theta)\left[f^{\prime}(\theta)\right]^{2} \alpha_{0}(\theta)+\frac{1}{2}\left[f^{\prime}(\theta)\right]^{2} \alpha_{1}(\theta)+\alpha_{3}(\theta), \\
\lim _{r \rightarrow \infty} r^{1 / 2}\left[\frac{\partial \tilde{\varphi}_{n 3}}{\partial r}-i \frac{\kappa_{n}}{H} \tilde{\varphi}_{n 3}\right]=0 .
\end{gathered}
$$

The third-order correction potential, $W_{3}(r, \theta)$, is given by

$$
W_{3}(r, \theta)=-\frac{H^{2}}{q} \sum_{n=-2}^{\infty} \frac{\tilde{\varphi}_{n 3}(r, \theta)}{Q_{n}} .
$$

The deflection (D.11) should satisfy the edge condition (65).

Let the second and the third terms on the right-hand side of (D.9) be presented by their Fourier series,

$$
\gamma_{2}(\theta)=\frac{1}{2}\left[f^{\prime}(\theta)\right]^{2} \alpha_{1}(\theta)-f(\theta)\left[f^{\prime}(\theta)\right]^{2} \alpha_{0}(\theta)=\sum_{m=0}^{\infty} \frac{\epsilon_{m}}{2}\left[\gamma_{2 m}^{(c)} \cos (m \theta)+\gamma_{2 m}^{(s)} \sin (m \theta)\right],
$$

then the boundary condition (D.9),

$$
\tilde{\varphi}_{n 3, r}(b, \theta)=\tilde{G}_{n 3}(\theta)+\gamma_{2}(\theta)+\alpha_{3}(\theta),
$$

provides the solution,

$$
\tilde{\varphi}_{n 3}(r, \theta)=\sum_{m=0}^{\infty} \frac{\epsilon_{m}}{2}\left\{\left[\tilde{G}_{n 3 m}^{(c)}+\gamma_{2 m}^{(c)}+\alpha_{3 m}^{(c)}\right] \cos (m \theta)+\left[\tilde{G}_{n 3 m}^{(s)}+\gamma_{2 m}^{(s)}+\alpha_{3 m}^{(s)}\right] \sin (m \theta)\right\} R_{n m}(r), \quad n \geq-2 .
$$

The relation (D.11) and the condition (65) give

$$
\begin{aligned}
& -\frac{H^{2}}{q} \sum_{n=-2}^{\infty} \frac{\tilde{\varphi}_{n 3}(b, \theta)}{Q_{n}}=\left[\Phi_{3}(b, \theta)+b f(\theta) \Phi_{2, r}(b, \theta)+\frac{[b f(\theta)]^{2}}{2} \Phi_{1, r r}(b, \theta)+\frac{[b f(\theta)]^{3}}{6} \Phi_{0, r r r}(b, \theta)\right] \\
& -b f(\theta)\left[-\frac{H^{2}}{q} \sum_{n=-2}^{\infty} \frac{\tilde{\varphi}_{n 2, r}(b, \theta)}{Q_{n}}\right]-\frac{[b f(\theta)]^{2}}{2}\left[-\frac{H^{2}}{q} \sum_{n=-2}^{\infty} \frac{\tilde{\varphi}_{n 1, r r}(b, \theta)}{Q_{n}}\right]-\frac{[b f(\theta)]^{3}}{6}\left[-\frac{H^{2}}{q} \sum_{n=-2}^{\infty} \frac{\tilde{\varphi}_{n 0, r r r}(b, \theta)}{Q_{n}}\right] .
\end{aligned}
$$

The right-hand side of (D.12) consists of summation and multiplication of Fourier series which together give a Fourier series,

$$
s_{2}(\theta)=\sum_{m=0}^{\infty} \frac{\epsilon_{m}}{2}\left[s_{2 m}^{(c)} \cos (m \theta)+s_{2 m}^{(s)} \sin (m \theta)\right]
$$

with known coefficients $s_{2 m}^{(c)}$ and $s_{2 m}^{(s)}, m \geq 0$. Then (D.12) can be written as

$$
\begin{aligned}
-\frac{H^{2}}{q} \sum_{m=0}^{\infty} \frac{\epsilon_{m}}{2}\left\{\left(\left[\gamma_{2 m}^{(c)}+\alpha_{3 m}^{(c)}\right] g_{m}^{-1}+\tilde{g}_{3 m}^{(c)}\right) \cos (m \theta)+\left(\left[\gamma_{2 m}^{(s)}+\alpha_{3 m}^{(s)}\right]\right.\right. & \left.\left.g_{m}^{-1}+\tilde{g}_{3 m}^{(s)}\right) \sin (m \theta)\right\} \\
& =\sum_{m=0}^{\infty} \frac{\epsilon_{m}}{2}\left[s_{2 m}^{(c)} \cos (m \theta)+s_{2 m}^{(s)} \sin (m \theta)\right]
\end{aligned}
$$

where $\tilde{g}_{3 m}^{(c)}$ and $\tilde{g}_{3 m}^{(s)}$ are defined in (79). Hence, 


$$
\begin{gathered}
\alpha_{3 m}^{(c)}=\left(-\frac{q}{H^{2}} s_{2 m}^{(c)}-\tilde{g}_{3 m}^{(c)}\right) g_{m}-\gamma_{2 m}^{(c)}, \quad m=0,1,2,3, \ldots, \\
\alpha_{3 m}^{(s)}=\left(-\frac{q}{H^{2}} s_{2 m}^{(s)}-\tilde{g}_{3 m}^{(s)}\right) g_{m}-\gamma_{2 m}^{(s)}, \quad m=1,2,3, \ldots
\end{gathered}
$$

